IAC-06-D5.2.05

\title{
RADIATION AND PLASMA ENVIRONMENTS FOR LUNAR MISSIONS
}

\author{
Joseph I. Minow and David L. Edwards \\ EV13/Natural Environments Branch \\ NASA, Marshall Space Flight Center, Huntsville, Alabama \\ joseph.i.minow@nasa.gov \\ Richard L. Altstatt, Anne M. Diekmann, and William C. Blackwell, Jr. \\ Jacobs, MSFC Group \\ NASA, Marshall Space Flight Center, Huntsville, Alabama \\ Katherine J. Harine \\ Raytheon ITSS, MSFC Group \\ NASA, Marshall Space Flight Center, Huntsville, Alabama
}

\begin{abstract}
Space system design for lunar orbit and extended operations on the lunar surface requires analysis of potential system vulnerabilities to plasma and radiation environments to minimize anomalies and assure that environmental failures do not occur during the mission. Individual environments include the trapped particles in Earth's radiation belts, solar energetic particles and galactic cosmic rays, plasma environments encountered in transit to the moon and on the lunar surface (solar wind, terrestrial magnetosheath and magnetotail, and lunar photoelectrons), and solar ultraviolet and extreme ultraviolet photons. These are the plasma and radiation environments which contribute to a variety of effects on space systems including total ionizing dose and dose rate effects in electronics, degradation of materials in the space environment, and charging of spacecraft and lunar dust. This paper provides a survey of the relevant charged particle and photon environments of importance to lunar mission design ranging from the lowest $(\sim$ few 10 's eV) photoelectron energies to the highest $(\sim \mathrm{GeV})$ cosmic ray energies.
\end{abstract}

\subsection{Introduction.}

Developing reliable space systems for exploration of the Moon and extended duration presence on the lunar surface requires analysis of potential system vulnerabilities to total ionizing dose, single event upsets, surface and bulk charging, and other effects on materials and systems due to exposure to the space radiation and plasma environment.

Plasma and radiation environments that will be encountered during lunar missions begin with the outbound and inbound trajectories through the Earth's radiation belts where spacecraft are exposed to trapped energetic ions which generate upsets in vulnerable electronic systems and energetic electron environments responsible for surface and bulk charging of spacecraft systems. Lunar orbital and surface charged particle environments are dominated at the highest flux levels by the relatively low energy solar wind with monthly encounters with the terrestrial magnetosheath and low density, hot plasma in the terrestrial magnetotail. Episodic energetic charged particle enhancements are also observed in both the lunar environment and in transit to the Moon from a variety of sources including solar energetic particle, events and particle enhancements in the Earth's foreshock region. Episodic energetic charged particle enhancements are also observed in both the lunar environment and in transit to the Moon from a variety of sources including solar energetic particle events, substorm events in the Earth's magnetotail, and particle enhancements in the Earth's foreshock region. These events may produce enhanced spacecraft charging environments and, in the case of the solar energetic particle events, may dominate the total ionizing dose for lunar missions. Solar energetic particle events are likely to be the radiation design drivers for short term lunar missions (less than a year) while the penetrating nature of galactic cosmic rays are a serious concern for human radiation dose when contemplating missions exceeding a few years in length. In addition to solar wind and the energetic solar and galactic particle sources, the daytime plasma environments near the lunar surface are dominated by photoelectrons emitted from interactions of solar UV/EUV photons with the lunar surface. Finally, the penetrating nature of galactic cosmic rays and the 


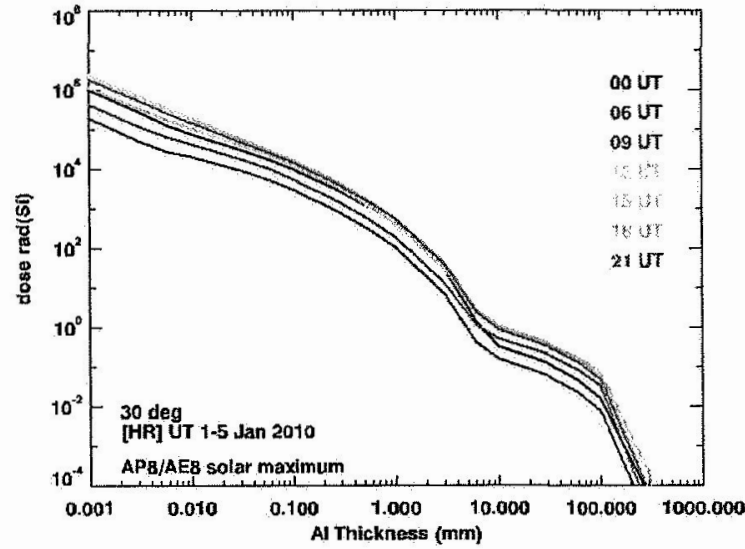

Figure 1. Departure Time Dependence for Translunar Injection Orbit Radiation Dose. Dose due to trapped electrons and ions during a single pass outbound through the Earth's radiation belts are given for a range of departure times.

highest energy solar energetic particle events are a serious concern for both human radiation dose and upset rates in electronic systems. This paper reviews the relevant plasma and radiation environments that must be considered in the design and operation of lunar missions.

\subsection{Terrestrial Radiation Belts}

Translunar injection and trans-Earth return trajectories require the vehicle to transit the radiation environments trapped within the Earth's magnetosphere. The amount of radiation encountered while traveling through the radiation belts depends on a number of factors including the latitude of the launch or landing site, the low Earth orbit departure or arrival longitude, geomagnetic activity and phase in solar cycle, and details of the individual orbit selected for the mission including orbit inclination and spacecraft velocity that will depend ultimately on the location of the landing site on the Moon and the orientation of the Moon's orbit plane relative to that of the Earth [c.f., Bate et al., 1971].

For example, Figure 1 shows radiation dose variations for single outbound $30^{\circ}$ inclination translunar injection orbits for a range of departure times throughout a single day. Dose is estimated from sampling the AE-8 trapped electron [Vette, 1991] and AP-8 trapped proton [Sawyer and Vette, 1976] radiation belt models along the $30^{\circ}$ inclination elliptical orbit. Dose in silicon as a function of depth in a semi-infinite aluminum shield is computed using the Shieldose-2 radiation transport code [Seltzer, 1980, 1994]. We have assumed an initial $300 \mathrm{~km}$ perigee low Earth orbit parking orbit with lunar phasing maneuver at $0^{\circ}$ right ascension of ascending node, $0^{\circ}$ argument of perigee, and $0^{\circ}$ true anomaly. Apogee is $379,867 \mathrm{~km}$ for a $100 \mathrm{~km}$ altitude lunar orbit and mean Earth-Moon distance of $384,400 \mathrm{~km}$ and the period of the orbit is approximately eight days with slightly more than 4 days required to travel from the Earth to the Moon. Departure times are adjusted by shifting the local time of the departure right ascension of ascending node.

Variations in radiation dose are within approximately an order of magnitude for the cases shown here and are due to the time dependent radiation belt encounter geometry for each of the orbits. Contributions from solar energetic particle events (which can be significant) and galactic cosmic rays (negligible) are not included and only the radiation dose from the electrons and ions trapped in the Earth's magnetic field are included in the estimate.

Direct co-planar transfer orbits are only possible when the translunar/trans-Earth injection orbit lies in the plane of the Moons orbit. The inclination of the lunar orbit varies from 18.2 to 28.5 degrees relative to the Earth's equator with a period of 18.6 years. Direct orbits originating from due east launches are possible only from launch sites at latitudes $\geq 28.5$ degrees latitude and other sites must generally use non-coplanar trajectories. For example, co-planar trajectories are possible from Kennedy Space Center at 28.5 degrees north latitude once every 18.6 years but higher inclination orbits must be used at other times.

The ten Apollo program flights which orbited the Moon (Apollo 8 through Apollo 17) all were launched from what is now the Kennedy Space Center (KSC) on the east coast of Florida at 28.5 degree north latitude. A direct $\sim 28.5$ degree inclination coplanar translunar injection orbit was only possible from KSC during the Apollo era on 25 March 1969 when the Moon was at an extreme north declination of $28^{\circ} 43^{\prime} 32^{\prime \prime}$ ' and additional dates [Meeus, 1997] when the coplanar trajectories are possible from KSC include 15 September 1987

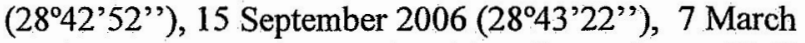
$2025\left(28^{\circ} 43^{\prime} 00^{\prime \prime}\right)$, and 25 September 2043 $\left(28^{\circ} 43^{\prime} 109^{\prime}\right)$. All other times the flight inclination will be greater than the minimum inclination obtained from a due east launch resulting in reduced radiation dose. Translunar injection orbit inclinations utilized for the Apollo flights ranged from a minimum of 28.5 degrees to a maximum of 32.55 degrees [Orloff, 2000]. More recently, the Lunar Prospector spacecraft (also launched from 28.5 degrees north latitude at Cape Canaveral Air Force Station) utilized a 29.2 degree inclination translunar injection orbit [Lozier et al., 1998]. In contrast, the Clementine spacecraft was launched from 


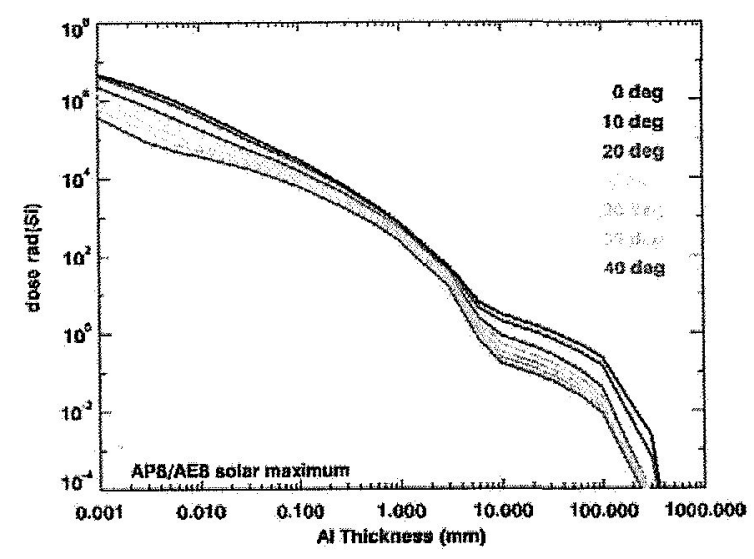

a)

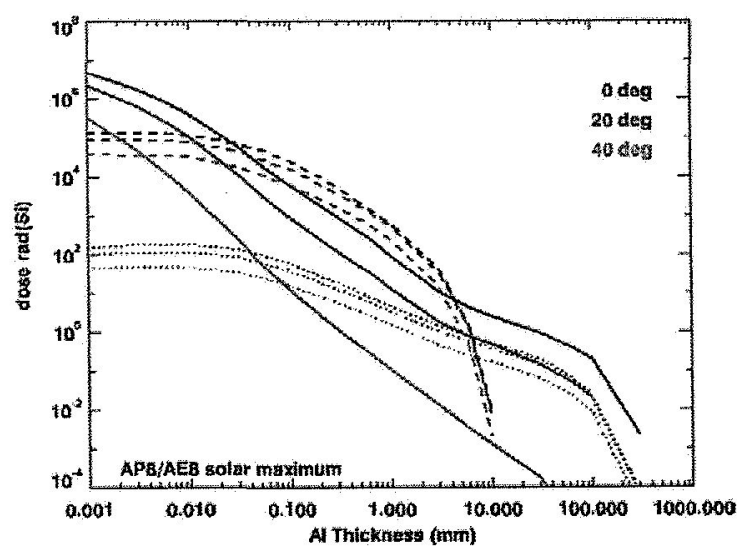

b)

Figure 2. Translunar Injection Orbit Radiation Dose. (a) Dose due to electrons and ions derived from the AE-8/AP-8 trapped radiation belt models for a single pass outbound pass through the Earth's radiation belts. (b) Dose contributions from protons (solid), electrons (dash), and bremstrahlung (dotted) for $0^{\circ}, 20^{\circ}$, and $40^{\circ}$ inclination translunar injection orbits.

Vandenburg Air Force Base at 34.75 deg north latitude into a lunar injection orbit inclined at 67 degrees [Regeon et al., 1994] and the European Space Agency Smart-1 spacecraft launched from the near-equatorial $5.05 \mathrm{deg}$ north latitude facility at Kourou, French Guiana, utilized a translunar injection orbit initially inclined 7degrees from the equator [ESA, 2003]. Finally, the series of Russian Luna probes launched in the 1960's and 1970's from Baikanur, Khazakstan, into near polar orbits with inclinations of 73 deg received some of the smallest radiation doses while traversing Earth's radiation belts.

Radiation dose for single outbound translunar injection orbits are shown in Figure 2-a for a range of inclinations. The same translunar injection elliptical orbit parameters used in the previous departure time example are used here including the $300 \mathrm{~km}$ perigee, $379,867 \mathrm{~km}$ apogee, lunar phasing maneuver at $0^{\circ}$ right ascension of ascending node, $0^{\circ}$ argument of perigee, and $0^{\circ}$ true anomaly. Total ionizing dose is obtained from the AE-8 and AP-8 solar maximum models and contributions from solar energetic particle events and galactic cosmic rays are not included. The greater the orbital inclination for the trajectory through the radiation belts, the smaller the radiation dose received by the spacecraft during transit of the radiation belts.

Figure 2-b shows the contributions from trapped electrons, electron bremstrahlung, and trapped protons to the total dose for three inclinations of the orbit used in the previous examples. Proton contributions dominate for very thin materials and very thick materials with electrons providing the dominant contributions at intermediate depths. Surface dose (depths $\leq 0.1 \mathrm{~mm}$ ) is most important for thermal control materials where surface damage modifies the optical properties. If the surface doses exceeding a few tenths of a megarad shown in Figure 1 prove to be of concern in some applications, it appears that increasing the inclination of the radiation belt transit orbits is an effective method of mitigating the issue.

Charging environments in the Earth's radiation belts are particularly harsh. For example, spacecraft surface charging in geostationary orbit may produce extreme potentials on the order of kilovolts in sunlight and 10's of kilovolts in darkness [Olsen, 1986] and bulk (deep dielectric) charging of insulating components and isolated conductors may lead to electrostatic discharge induced anomalies or even failures [Koons et al, 1998].

Figure 3-a from Garrett and Hoffman [2000] provide estimates of spacecraft potentials for a spherical spacecraft in darkness as a function of altitude and latitude in the Earth's magnetosphere demonstrating where extreme spacecraft potential values may be observed due to surface charging. Fortunately the values are significantly reduced when the spacecraft is exposed to sunlight (due to the emission of photoelectrons which reduces the amount of charge accumulated on the spacecraft). Extreme charging conditions in the midnight sector under eclipse conditions which must be endured by spacecraft in geostationary orbit is easily mitigated for lunar missions by judicious orbit selection. 


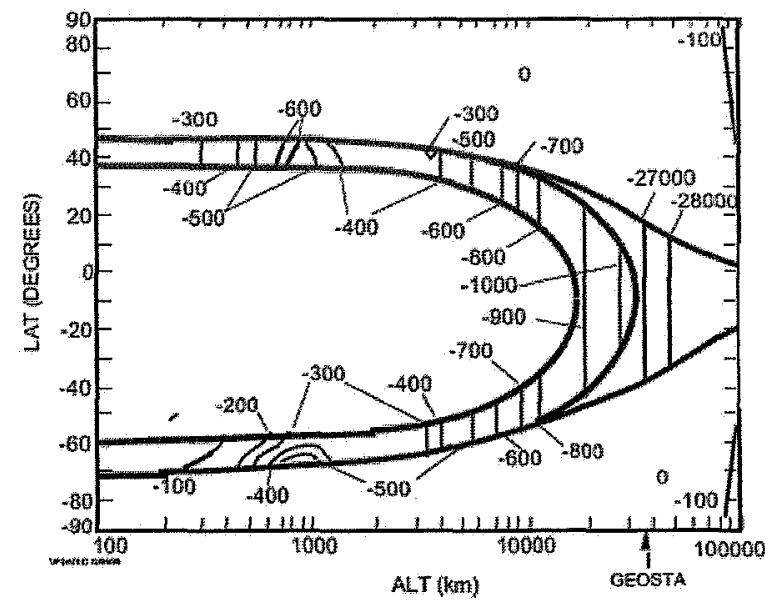

a)

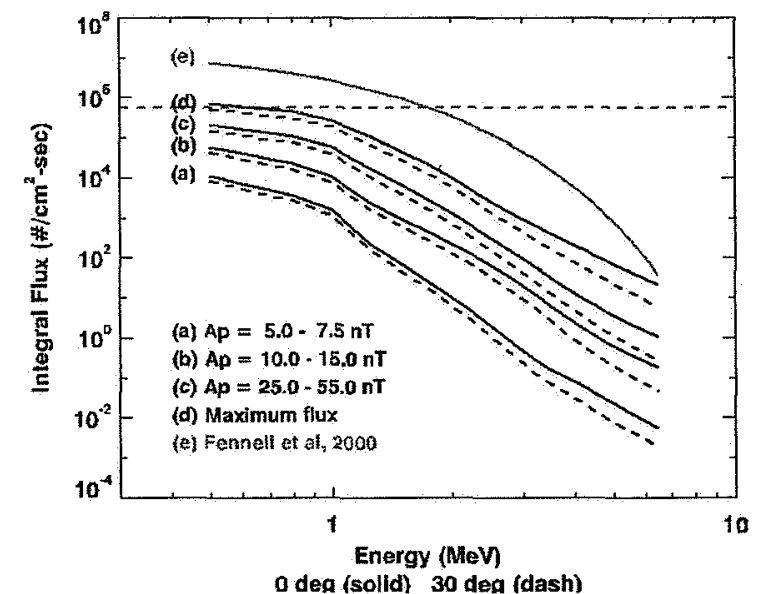

b)

Figure 3. Terrestrial Spacecraft Charging Environments. (a) Surface charging potentials in darkness due to collection of currents from magnetospheric plasma environments (from Garrett and Hoffman (2000). (b) Bulk charging environments for 0 degree and 30 degree inclination translunar injection orbits are compared to a worst case bulk (deep dielectric) charging design environment [Fennell et al., 2000].

Figure 3-b gives orbit averaged $0.5 \mathrm{MeV}<\mathrm{E}<6 \mathrm{MeV}$ electron flux environments generally associated with bulk (deep dielectric) charging computed for hypothetical orbits with perigee and apogee at $300 \mathrm{~km}$ and $379,870 \mathrm{~km}$, respectively, assuming the vehicle is inserted into a $100 \mathrm{~km}$ lunar altitude orbit. The CRRESELE trapped electron model [Brautigam and Bell, 1995] provides the electron environments and orbit averaged integral electron flux values are shown for increasing geomagnetic activity levels in each of the curves (a) through (d) where the magnitude of geomagnetic disturbances are indicated by the planetary $A_{p}$ index. Modeled average electron flux is a maximum for 0 degree inclination orbits (solid curves) while the average electron flux is reduced (dashed curves) for 30 degree inclination orbits (consistent with inclinations used for the Apollo flights).

The final curve (e) in Figure 3-b is the orbit average flux for half of a 0 degree lunar phasing orbit reported by Fennell et al. [2000] as a preliminary worst case bulk (deep dielectric) charging design environment for lunar orbits derived from Combined Release and Radiation Effects Satellite (CRRES) satellite energetic electron measurements. Fennell et al. [2000] developed the bulk charging environment specification by mapping CRRES data from the spacecraft's 18 degree inclination geostationary transfer orbit to the geomagnetic equator and computing the orbit averaged electron flux for a 0 degree inclination, 10 hour lunar phasing orbit centered on perigee. The phasing orbit therefore included both the inbound and outbound segments of the lunar phasing orbit through the Earth's radiation belts with apogee near lunar orbit and perigee in low Earth orbit and the flux is reduced by a factor of two assuming the most common mission profiles for future lunar missions will utilize direct insertion translunar injection trajectories.

A blue dashed line indicates the flux threshold of $5.56 \times 10^{5} \mathrm{e}^{-} / \mathrm{cm}^{2}-\mathrm{sec}$ flux corresponding to the 10 hour fluence $2 \times 10^{10} \mathrm{e}^{-} / \mathrm{cm}^{2}$ electron fluence identified as the threshold where pulsing in insulators exposed to energetic electrons begins, to be observed in geostationary transfer orbits [NASA-4002, 1999]. Insulators on the surface of the spacecraft or lightly shielded materials may be charged by $0.1 \mathrm{keV}$ to $1 \mathrm{MeV}$ electrons but only at the highest geomagnetic disturbance levels. Electrons $>1 \mathrm{MeV}$ which penetrate significant thickness of shielding appear to only be a bulk charging issue on approach to or return from the Moon at the most extreme environments for orbits near the equatorial plane while serious issue with bulk charging are not expected except for the most lightly shielded materials.

It should be noted that the $2 \times 10^{10} \mathrm{e}^{-/} \mathrm{cm}^{2}$ electron fluence accumulated in 10 hours threshold assumes some values typical of insulator conductivity and temperatures observed in geostationary transfer orbit satellites giving time constants on the order of 10 hours or less for charge storage in the insulating materials. Threats for charging may occur at lower electron flux levels if the insulators are very cold because insulator electrical resistivity increases with decreasing 


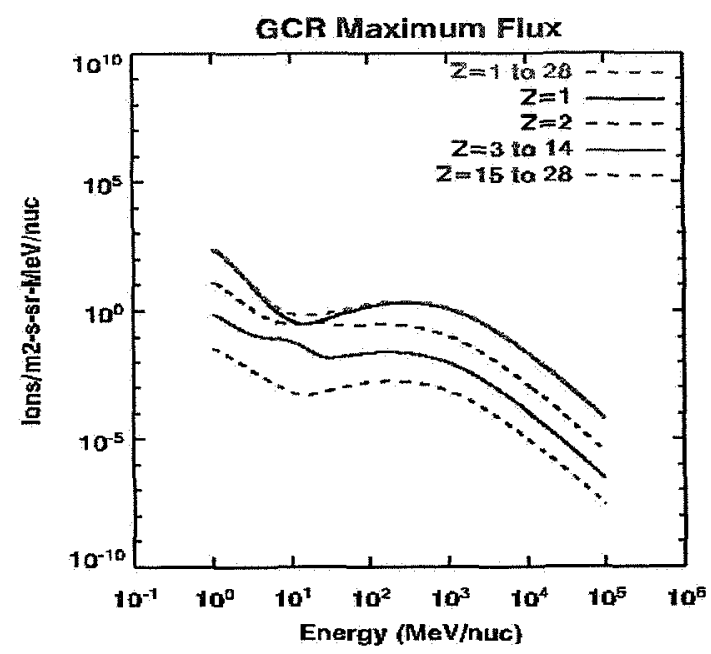

a)

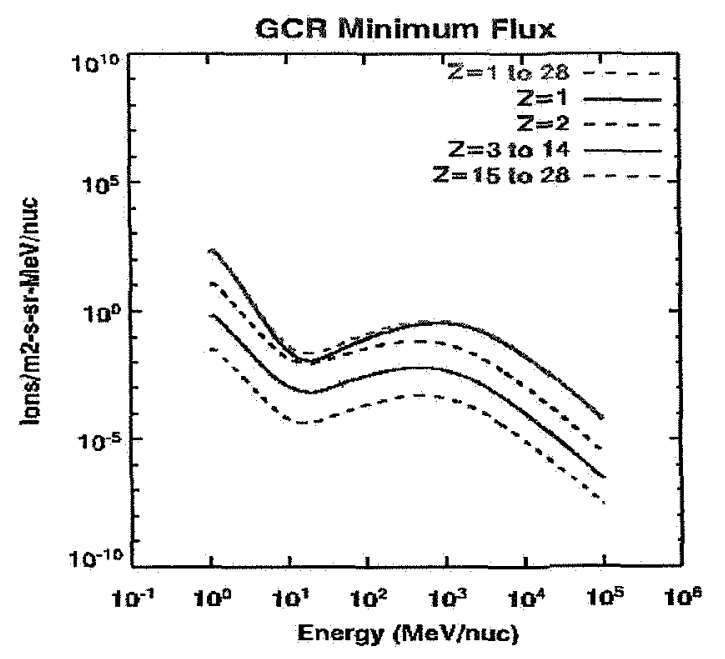

b)

Figure 4. Galactic Cosmic Rays. (a) Solar minimum conditions provide the maximum GCR flux while (b) solar maximum conditions yield a minimum GCR flux.

temperature and the bulk charging time constant increase to very long periods allowing charge densities to accumulate in insulators for periods longer than ten hours. Future goals in lunar exploration may include low temperature regions which are still exposed to electron charging environments such as the dark side of the moon where temperatures drop to $100 \mathrm{~K}$ during the two week lunar night or the lunar poles where permanently shadowed craters may reach temperatures of $40 \mathrm{~K}$. Further evaluation of space systems designed for operation in these environments for potential bulk charging issues is certainly warranted.

\subsection{Galactic Cosmic Rays and Solar Particle Events}

Galactic cosmic rays (GCR) are electrons and ions accelerated to extremely high energies in astrophysical processes outside of the solar system. The GCR energy spectrum (both intensity and particle energy) is reduced as the particles penetrate into the heliosphere due to scattering by irregularities in the interplanetary magnetic field [Parker, 1965; Fillius and Axford, 1985; Badhwar and O'Neill, 1996]. Because the magnetic field irregularities are solar cycle dependent with enhanced irregularities occurring during solar maximum, the GCR flux spectra exhibits a solar cycle modulation. GCR flux varies over a factor of approximately 2.5 from solar minimum to solar maximum with the greatest GCR flux observed at solar minimum. Flux variations are shown in Figure 4 for protons $(Z=1)$, helium $(Z=2)$, the summed flux for Li to Si $(3 \leq Z \leq 14)$, and the summed flux for $P$ to $\mathrm{Ni}(15 \leq Z$ $\leq 28$ ). GCR flux is obtained from the 1996 version [Tylka et al., 1997] of the Cosmic Ray Effects on
Microelectronics (CREME96) model [Adams, 1986] which includes not only the primary GCR component which dominate the spectrum at energies greater than $\sim 50 \mathrm{MeV} / \mathrm{nuc}$, but anomalous cosmic ray ions at energies of $\sim 10-50 \mathrm{MeV} / \mathrm{nuc}$ (during solar minimum) and a low energy solar component which dominates at energies less than $\sim 10 \mathrm{MeV} /$ nuc. While cosmic ray fluence is not generally considered a significant source for ionizing dose in materials compared to the much larger particle fluence accumulated during solar energetic particle events, the GCR heavy ion component are a significant source of energetic ions producing single event effects in electronic systems and a primary concern for biological systems due to the penetrating nature of the radiation.

Cosmic ray flux is isotropic at $1 \mathrm{AU}$ so the dose to the surface can be easily estimated by multiplying the differential flux by a factor of approximately obtained three from integrating the solid angle over the full range of azimuthal angles and the polar angle from zenith to the horizon. Since the full flux to a point is $4 \mathrm{pi}^{*} \mathrm{Jo}_{\mathrm{o}}$, the shielding afforded by the Moon for lunar surface operations is $1 / 4$ the flux observed in free space.

Transient disturbances in the Sun's outer atmosphere including flares and coronal mass ejections are a source of energetic ion fluxes in interplanetary space. The most intense solar energetic particle (SEP) events are produced by ion acceleration at the expanding shock front of coronal mass ejections and are the source of the most intense energetic radiation environments in interplanetary space. SEP events occur sporadically with the greatest probability in the years of solar maximum and for the first few years after the peak of 


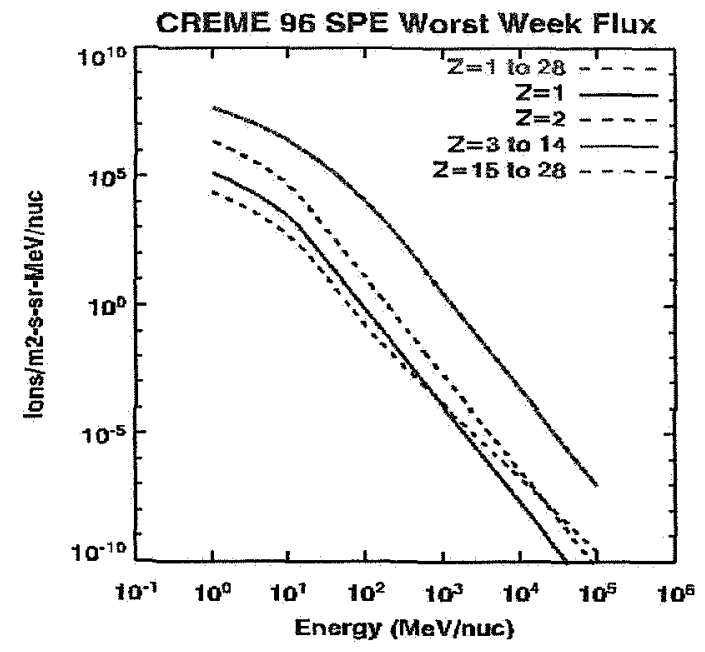

a)

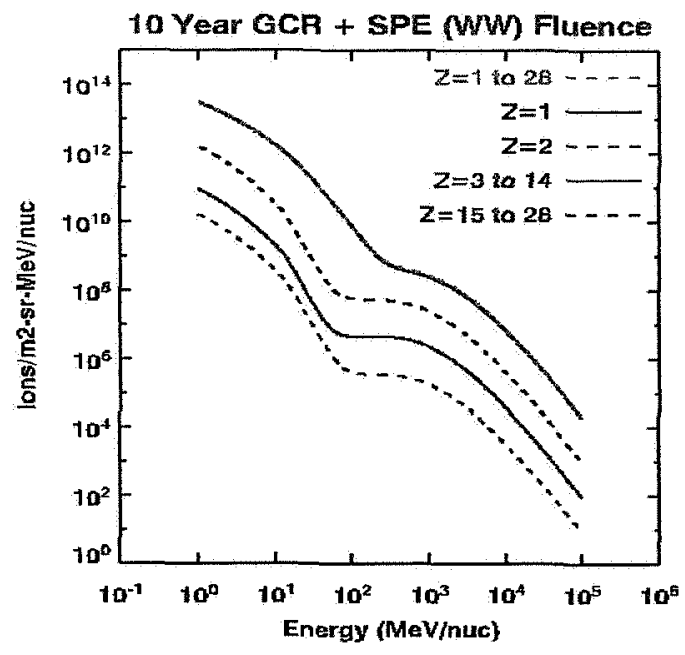

b)

Figure 5. Solar energetic particles and 10 year fluence environment.

(a) CRÈME96 worst week average flux. (b) Ion fluence environment derived from 5 year GCR solar maximum ion fluence, 5 year GCR solar minimum ion fluence, and the 7.5 days fluence of the worst week solar proton average flux given in (a).

solar cycle. Individual events may last more than a week in extreme cases, but the effects typically last hours to days. Predicting individual SEP events is difficult, but it can be assumed that missions active over a solar cycle or longer will encounter a number of events during solar maximum and at least one large SEP per solar cycle. Engineering design for materials exposed to SEP radiation environments generally required qualifying material properties to remain within specified end of life values for at least one large SEP per solar cycle.

The 1996 version of the Cosmic Ray Effects on Microelectronics (CREME96) provides SEP "worst week" flux, "worst-day" flux, and "peak flux" models derived from observations during the October 1989 SEP events [Tylka et al., 1997] for use in evaluating radiation effects on space systems and effectiveness of shielding for protection against the most severe interplanetary radiation environments. The WorstWeek Model (Figure 5-a) gives the average flux over a 7.5 day interval (starting at 1300 UT on 19 October 1989). CREME96 worst week model is an extreme model of an event which would occur only once during a solar cycle, or approximately a period of nine to eleven years. Although as many as fifty SEP events may occur during a solar cycle, the fluence from the sum of many individual, small events may be dominated by the contribution of a single large SPE event. It is traditional in the space systems engineering community to include the fluence from a single worst case event over a solar cycle for SEP event environment for this reason. Large events contain significant quantities of charged particles with energies in excess of $10 \mathrm{MeV}$, which will generate dose even in shielded materials.

The one year lunar surface radiation environment provided in Figure 5-b is derived from the solar energetic ion fluence provided by the CREME96 WorstWeek Model flux over a 7.5 day period with a one year GCR fluence contribution (solar maximum). Ion fluence spectra are given for hydrogen $(Z=1)$, helium $(Z=2)$, summed $\mathrm{Li}$ to $\mathrm{Si}(3 \leq \mathrm{Z} \leq 14)$ fluence, and summed $\mathrm{P}$ to $\mathrm{Ni}(15 \leq \mathrm{Z} \leq 28)$ fluence.

\subsection{Energetic Particle Dose for Lunar Missions}

An estimate for the radiation dose to materials appropriate for a one year lunar mission can be estimated for the radiation belt transit and solar energetic proton environments described in the previous sections. The same $300 \mathrm{~km}$ perigee low Earth orbit parking orbit with lunar phasing maneuver at $0^{\circ}$ right ascension of ascending node, $0^{\circ}$ argument of perigee, $0^{\circ}$ true anomaly and $379,867 \mathrm{~km}$ apogee is used with a $30^{\circ}$ inclination for the lunar transfer orbit. Trapped particle environments are obtained once again from the $\mathrm{AE}$ 8/AP-8 models but contributions from solar energetic particle events are included as well. The Emission of Solar Proton (ESP) model [Xapsos, 1999] provides the solar energetic proton fluence. Two confidence levels 


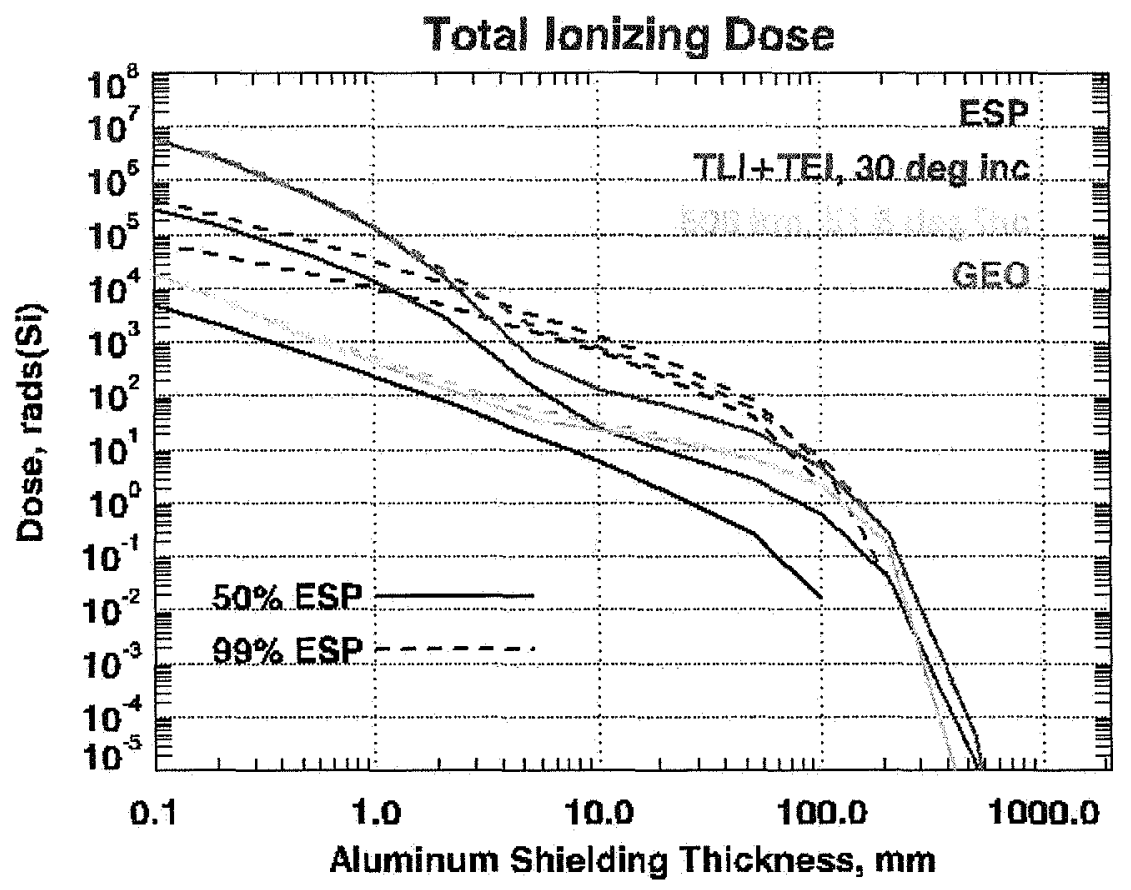

Figure 6. Lunar Energetic Particle 1-Year Mission Dose for Materials. Dose due only to 50\% (solid) and 99\% (dash) ESP solar proton environments are shown in black. Blue lines are the trapped radiation belt dose contributions from $30^{\circ}$ inclination translunar (TLI) and trans-Earth (TEI) injection orbits including contributions from the $50 \%$ and $99 \%$ solar proton environments. Geostationary orbit (red) and $500 \mathrm{~km}, 51.6$ low Earth orbit (green) examples including both trapped and $50 \%, 99 \%$ solar proton events are shown for comparison.

are adopted here, a $50 \%$ level for mean environments and a $99 \%$ confidence level to estimate extreme environments due to large solar energetic particle events.

Two additional total dose environments are included for comparison. A one year dose from a low Earth orbit $500 \mathrm{~km}, 51.6^{\circ}$ inclination orbit consistent with the ISS Ionizing Radiation Design Environment [SSP-30512] includes contributions from the trapped protons and electrons as well as $50 \%$ and $99 \%$ ESP solar protons shielded by the Earth's geomagnetic field. There is little difference in the two dose environments due to the effective shielding provided by the terrestrial magnetic over most of the ISS orbit. The geostationary orbit environment is unshielded by the Earth's geomagnetic field to solar energetic particles but appears to exhibit little variation due to the $50 \%$ and $99 \%$ solar proton contributions. The apparent discrepancy is resolved by noting that the trapped electron environments dominate the dose in materials over a wide range of shielding depths at geostationary orbit and proton dose is only significant over a restricted range of shielding depths.
These material results shown in Figure 6 are valid for materials with thickness less than approximately $50 \mathrm{~cm}$ where additional dose due to secondary particles begins to be important for crew dose issues. The total radiation dose is very small at the shielding thickness values included here and is not important for materials.

Estimates of dose due to galactic cosmic rays and solar ions with energies exceeding a few 10 's $\mathrm{MeV}$ require the use of radiation transport model. which include the generation of secondary particles due to nuclear interactions of the primary flux with shielding materials. Figure 7 provides incident galactic cosmic ray flux incident on varying thickness of Apollo-16 soil and proton and neutron flux emerging from the shielding material. These results are from the FLUkuierende KAskade (FLUKA) Monte Carlo radiation transport model [Fassò et al., 2001a,b; Fassò et al., 2003; Fassò et al., 2005] which includes the physics for ion nuclear reactions with shielding materials. Proton flux at energies less than approximately $10 \mathrm{MeV}$ are reduced by the shielding but once the incident particle energy exceeds $\sim 10 \mathrm{MeV}$ the transmitted flux is within an order of magnitude of the incident flux. The reason for this behavior is twofold. First, the reaction cross sections 

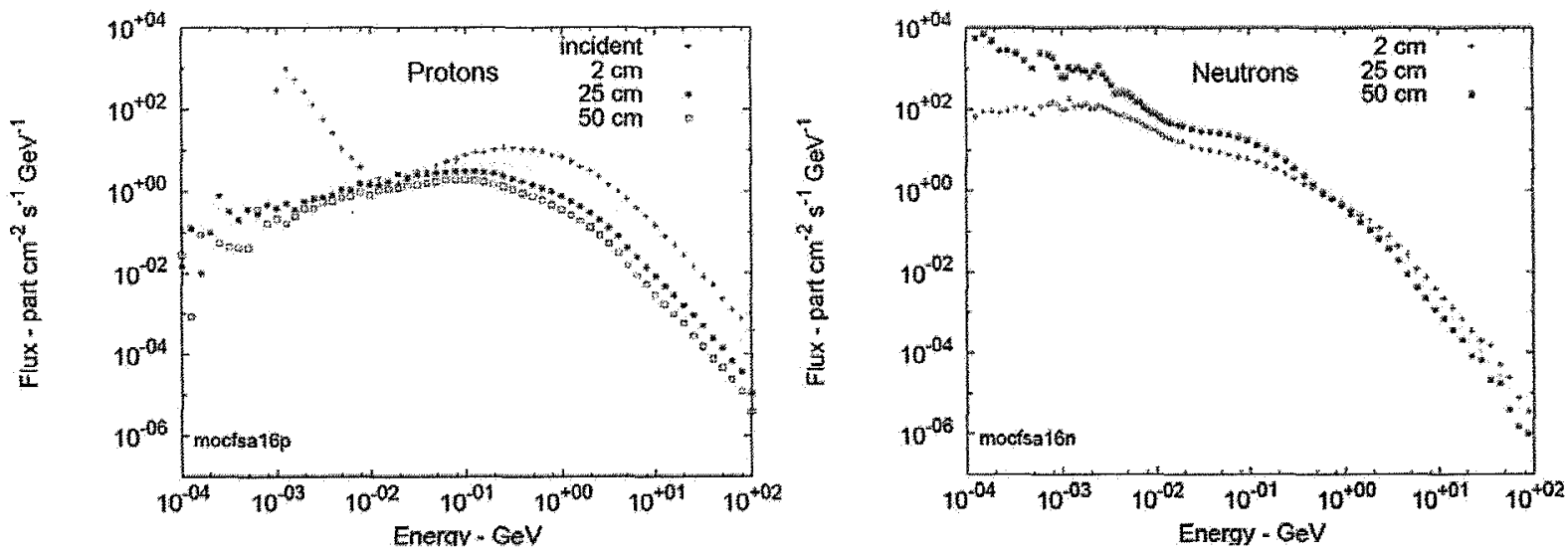

a)

b)

Figure 7. GCR Protons on Apollo-16 Soil. (a) Incident protons and transmitted protons including secondary protons generated in shielding material. (b) Neutrons emerging from shield generated by nuclear interactions of incident proton flux with Apollo-16 regolith.

are strongly energy dependent and small for large energies so the energetic protons can penetrate appreciable thickness of shielding. Second, inelastic interactions of cosmic ray particles with shielding materials at energies greater than a few tens $\mathrm{MeV}$ are capable of producing secondary particles. The proton flux emerging from the shielding material is therefore composed of both incident particles which penetrate the shield as well as secondary protons generated within the shield. Figure 7-b provides the neutron flux emerging from the Apollo-16 soil shield generated by proton interactions with the shielding material.

The FLUKA model treats physics of heavy ion interactions in addition to the protons. Figure 8 is an example of the radiation dose in silicon due to galactic cosmic ray ions from $Z=1$ to $Z=28$ as a function of shielding depths in Apollo-16 soil. The spectrum of the incident isotropic solar minimum cosmic ray ion environment is the CREME96 model solar minimum galactic cosmic ray spectrum shown in Figure 4-b. Dose for each of the $Z_{n}$ ions where $n=1,2, \ldots, 28$ is the total dose produced by the incident ion $Z_{\mathrm{n}}$ and all ions from $\mathrm{z}=1$ to 28 generated by interactions within the shield. Dose is dominated by the light ions $(\mathrm{H}, \mathrm{He})$ but significant peaks occur for heavy ions with $Z=6,7,8$ $(\mathrm{C}, \mathrm{N}, \mathrm{O}), \mathrm{Z}=12,13(\mathrm{Mg}, \mathrm{Si})$, and $\mathrm{Z}=26(\mathrm{Fe})$.

Integrated dose in silicon for all ion species in Figure 8 as a function of depth in Apollo-16 soil is given in Table 1. Space rated electronics parts are typically qualified for total ionizing radiation dose environments on the order of $10 \mathrm{~Gy}(1 \mathrm{krad})$ so the fractional total dose environments in Table 1 from galactic cosmic rays are negligible for electronics parts.
Wilson et al. [1997] results using the HZETRN deterministic transport code for solar minimum galactic cosmic ray dose in biological materials including skin, ocular lens, and blood forming organs as a function of depth in aluminum slab shielding is approximately $25 \%$ higher than the FLUKA results for dose in silicon. The results are comparable within an order of magnitude consistent with the differences in density of the target materials and differences in the galactic cosmic ray environments used for inputs to the codes.

\subsection{Plasma (Solar Wind, Magnetosheath, and Magnetotail) Environments}

Solar wind plasma energies are too low to produce bulk

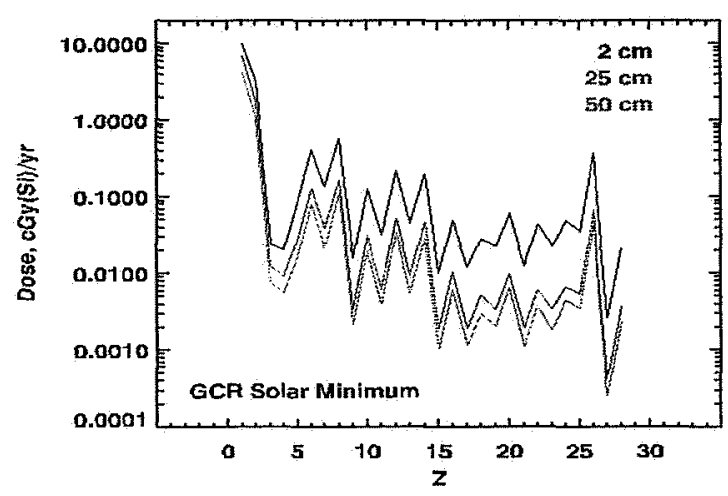

Figure 8. GCR Dose in Silicon as Function of Depth in Apollo-16 Soil. Dose for each of the $Z_{\mathrm{n}}, \mathrm{n}=1,2, \ldots 28$ ions is due to the incident ion and all ions $(1 \leq Z \leq 28)$ generated by the nuclear interactions within the shielding material 
Table 1. GCR Dose in Silicon Shielded by Lunar Regolith

\begin{tabular}{|c|c|c|}
\hline $\begin{array}{l}\text { Shieldi } \\
\text { (mm) }\end{array}$ & $\begin{array}{l}\text { gepth } \\
\left(\mathrm{g} / \mathrm{cm}^{2 *}\right)\end{array}$ & $\begin{array}{l}\text { Dose (Si) } \\
\text { (cGy/yr) }\end{array}$ \\
\hline 20 & 5.8 & 15.88 \\
\hline 250 & 72.5 & 9.27 \\
\hline 500 & 145.0 & 5.65 \\
\hline
\end{tabular}

damage in materials but may degrade surfaces properties of thermal control coatings (including optical properties required for heat rejection) and material integrity of thin materials used for thermal control systems on lunar orbiters and landers, regolith "sandbags," or other lunar infrastructure design features.

Lunar orbit is inclined approximately 5.1 degrees from the ecliptic plane yielding a maximum distance ranging \pm 5.3 Re that the Moon can be located from the plane. The magnetotail is approximately circular in cross section at lunar distances and the radius of the magnetopause (the boundary between the magnetotail and the magnetosheath) ranges from 20 to $30 \mathrm{Re}$ [Howe and Binsack, 1972]. The magnetotail is aligned with the solar wind, approximately 4 degrees from the Sun Earth line in the plane of the ecliptic, so the Moon must past through the magnetotail once each month. Bow shock dimensions are even larger, with the mean radius varying from $40 \mathrm{Re}$ to $70 \mathrm{Re}$ (or more) at lunar orbit [Bennett et al., 1997]. Using values of $\mathrm{R}_{\mathrm{MP}}=25 \mathrm{Re}$ and $\mathrm{R}_{\mathrm{BS}}=50 \mathrm{Re}$ for the magnetopause and bow shock radii, respectively, the fraction the lunar orbit inside the bow shock is $26.5 \%$, the fraction inside the magnetotail is $13.2 \%$, and the fraction in the magnetosheath (between the bow shock and magnetopause) is $13.3 \%$. The balance of the orbit, $73.5 \%$, is spent in the solar wind.

Free field plasma environments shown in Figure 9 appropriate for lunar distances are based on reconstructed differential flux distributions derived from mean solar wind [Feldman et al., 1977], magnetosheath, and plasma sheet [Minow et al. [2000] moment statistics using the a technique described elsewhere for reconstructing L2 and solar wind environments [Blackwell et al., 2000; Minow et al., 2004a, 2004b, $2005,2006]$. Electron and ion fluence environments for a ten year period are estimated using the fraction of an orbit the Moon spends in the solar wind $(73.5 \%)$, the magnetosheath (13.3\%), and the magnetotail (13.2\%) to scale the number of individual spectra included from each region when integrating reconstructed differential flux spectra over one lunar orbit to obtain the fluence. Magnetotail encounters are all assumed to be plasma sheet environments which adds a conservative high energy environment appropriate for engineering design although many magnetotail encounters are likely to be the low flux, lower energy lobe environments above or below the plasma sheet. The electron environments shown in Figure 9-a is a power law dominated by high flux at low energies because the component electron spectra are dominated by the high flux, low energy core component of the velocity distributions with smaller contributions from the halo and superhalo components. The ion fluences in Figure 9-b exhibit two peaks. The lower energy peak near $1 \mathrm{keV}$ is due to protons and higher energy peak due to helium ions.

Plasma environments near the moon are perturbed when the Moon passes through the Earth's magnetotail or a spacecraft passes behind the Moon. The effect of the Earth's magnetotail is demonstrated first in Figure 10-a where spin averaged electron flux records from the Lunar Prospector Electron Reflectometer instrument are shown for the month of April, 1998. The electron flux depletion at low electron energies for nearly five days before the middle of the month is the reduced plasma flux in the Earth's magnetotail. In addition, the effects of a solar energetic particle event is observed starting on 20 April 1998.

High time resolution Electron Reflectometer records over a two day period are shown in Figure 10-b demonstrating the modulation of the electron flux when the spacecraft passes through the lunar wake. Reductions in flux occur at approximately two hour intervals, the orbital period of the Lunar Prospector spacecraft, due to passage of the spacecraft through the solar wind wake. Reductions in electron flux occur at all energies but are the greatest for the low energy electrons.

\subsection{Lunar Photoelectrons and Surface Potentials}

Photoelectrons generated when solar ultraviolet (UV) and extreme ultraviolet (EUV) photons interact with the lunar surface. Since the photoelectron current is generally greater than the incident solar wind electron currents, the lunar surface will charge positive a few tens of volts [Manka and Michel, 1973; Freeman et al., 1973; Freeman and Ibrahim, 2004]. Photoelectron densities in the lunar plasma sheath have been reported to range from $130 \mathrm{e}-/ \mathrm{cm} 3$ at the lunar surface [Feuerbacher et al., 1972] to peaks on the order of 500 to $1000 \mathrm{H} / \mathrm{cm} 3$ at altitudes of $5-10 \mathrm{~km}$ above the lunar surface [Vyshlov, 1974; Vyshlov and Savich, 1978] although the in-situ observations obtained during the Apollo 12, 14, and 15 yielded photoelectron densities as large as $1 \times 10^{4} \mathrm{e} / \mathrm{cm}^{3}$ up to altitudes of $100 \mathrm{~m}$ [Reasoner and Burke, 1972]. 


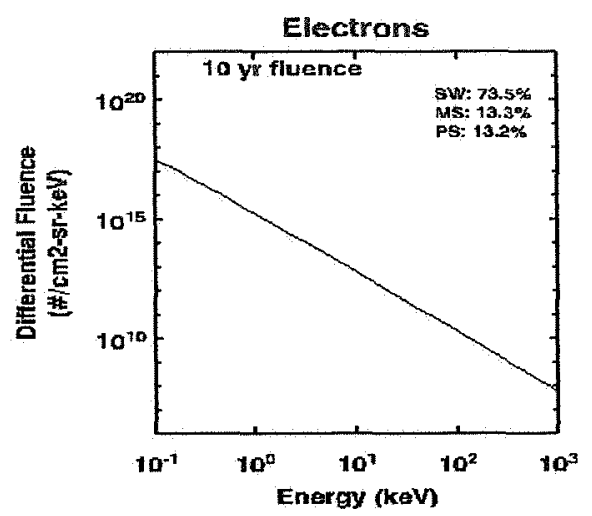

a)

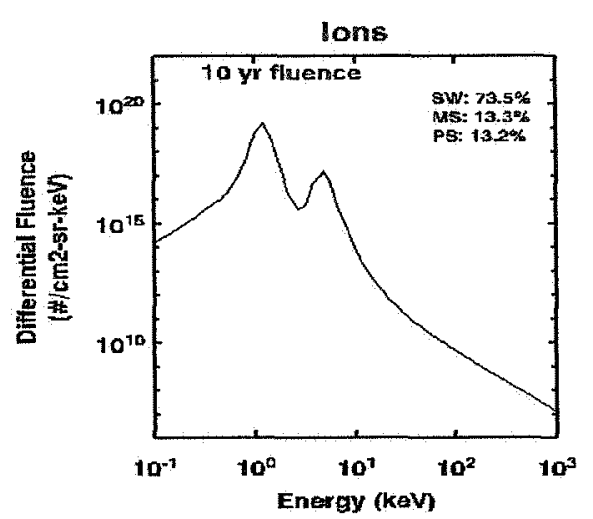

b)

Figure 9. Free-Field Lunar 10 Year Plasma Fluence. (a) Electron and (b) ion fluence environment for sunward facing surfaces of the lunar surface assuming the Moon spends $73.5 \%$ of the time in the solar wind, $13.3 \%$ of the time in the magnetosheath, and $13.2 \%$ of the time in the magnetotail (taken to be plasma sheet).

In contrast to the positive potentials which the lunar surface charges on the dayside, the terminator and nightside regions of the Moon which are not illuminated but is still exposed to energetic electrons arriving from the distant tails and sunward flowing components of the solar wind will charge to negative potentials [Knott, 1973; Mall and Borisov, 2001; Halekas et al., 2002]. WIND spacecraft measurements of wake structure on nightside suggests large negative potentials on the order of $-400 \mathrm{~V}$ in lunar plasma wake [Ogilvie et al., 1996] and measurements of negative lunar potentials are reported ranging from 10 to $100 \mathrm{~V}$ negative near the terminator [Lindeman et al., 1973; Freeman and Ibrahim, 2004] to values of $-300 \mathrm{~V}$ in the depth of the lunar wake [Halekas et al., 2005].

\subsection{Solar Photon Environments}

Solar ultraviolet (UV) and extreme ultraviolet (EUV) photons are sufficiently energetic to ionize materials and are a particular concern for degradation of organic compounds that may be used in lunar infrastructure design (for example, epoxy fillers for regolith blocks, polymer radiation shields, polymer "regolith bags" etc.). Effects will be restricted to surface and subsurface materials within a few optical depths at the wavelength of interest.

The solar photon ten year fluence environment given in Figure 11 is derived from the SOLAR2000 model [Tobiska et al., 2000; Tobiska, 2004] which provides daily photon flux spectra (photons/cm2-sec) scaled by solar activity. A ten year period from 1 January 1990 to 1 January 2000 was arbitrarily chosen to obtain the ten year photon fluence. This period starts with the peak of Solar Cycle 22, includes solar minimum between Cycle's 22 and 23, and the first peak in Cycle 23 yielding nearly a complete solar cycle.

\subsection{Discussion and Summary}

The plasma environments described in previous sections will charge spacecraft due to differential collection of currents from the space environment. As noted in Section 2.0, spacecraft potentials in transit to the Moon could reach kilovolt levels if the spacecraft passes through eclipse regions. Fortunately, this condition will likely to be rare for most spacecraft in transit to and returning from the Moon. However, even spacecraft in lunar orbit can charge to potentials on the order of hundreds of volts negative so evaluation of potential charging threats is important for design of spacecraft lunar exploration.

The radiation environments are not particularly challenging compared to those regularly encountered when designing spacecraft for long term use in geostationary orbit, geostationary transfer orbit, medium Earth orbit, or other orbits within the Earth's magnetosphere which regularly encounter the trapped flux within the Earth's magnetic field. The greatest challenge for lunar missions like likely be human radiation dose for long term missions on the Moon if inadequate shielding is provided to reduce the flux of primary cosmic rays and the secondary particles generated within shielding. However, even the cosmic ray environments are unlikely to represent a major concern for short term missions to the Moon. Spacecraft charging environments within the Earth's magnetosphere can be avoided using high inclination orbits or mitigated with good spacecraft design. Charging environments in lunar orbit regularly produce 


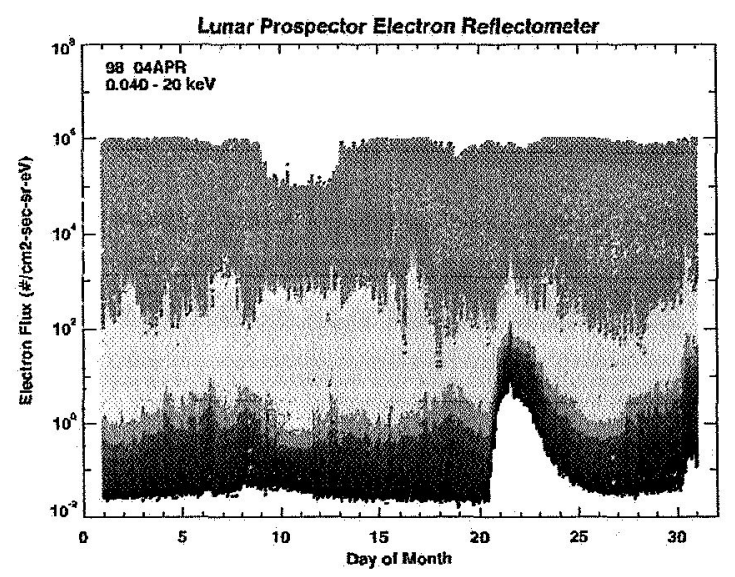

a)

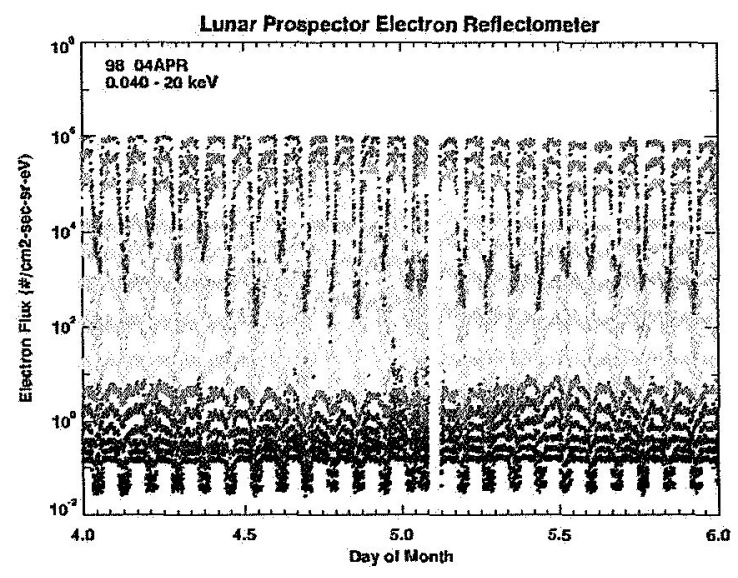

b)

Figure 10. Lunar $<\mathbf{2 0} \mathrm{keV}$ Electron Environments. Lunar Prospector Electron Reflectometer spin averaged $\sim 40 \mathrm{eV}$ (red) to $20 \mathrm{keV}$ (black) electron flux measurements from lunar orbit. (a) The complete month of April 1998 is shown including a depletion of the low energy electrons by nearly two orders of magnitude near the middle of the month during the period the Moon is inside the Earth's magnetotail. (b) Detail of electron flux variations for 4 to 6 April 1998 while the Moon is in the solar wind showing plasma depletions in the lunar wake.

negative potentials on the order of hundreds of volts suggesting the most challenging charging environments for lunar exploration will be the dark side of the Moon and craters which are bathed in plasma fluxes but are not illuminated by sunlight. Further evaluation of these regions is required to determine the extent of charging issues which may exist in dark lunar craters.

\section{Acknowledgements}

Lunar Prospector Electron Reflectometer data was provided courtesy of Dr. Raymond Walker, UCLAIGPP.

\section{References}

Adams, Jr., J.H., Cosmic Ray Effects on MicroElectronics (CREME), Part IV, Naval Research Laboratory Memorandum Report 5901, December 31, 1986.

Badhwar, G.D., and P.M. O'Neill, Galactic cosmic radiation model and its applications, Adv. Space Res., 17, 7-17, 1996.

Bate, R.R., D.D. Mueller, and J.E. White, Fundamentals of Astrodynamics, Dover Publications Inc., New York, 1971.

Bauer, S.J., Limits to a lunar ionosphere, Anzeiger Abt. II, 133: 17-21, 1996.

Bennett, L., M. G. Kivelson, K. K. Khurana, L. A. Frank, and W. R. Paterson, A model of the Earth's distant bow shock, J. Geophys. Res., 102, 26927 26941, 1997.

Blackwell, W.C., J.I. Minow, S.W. Evans, D.M. Hardage, and R.M Suggs, Charged Particle Environment Definition for NGST: Model Development, Proc, SPIE, 4013, 908-919, UV, Optical, and IR Space Telescopes and Instruments VI, J.B. Breckinridge and P. Jakobsen, eds., 2000.

Brautigam, D. H., and J. T. Bell, CRRESELE Documentation, PL-TR-95-2128, Environmental Research Papers, 1178, Phillips Laboratory, 1995.

ESA 2003, SMART-1 leaves Earth on a long journey to the Moon, ESA Press Release Number 60-2003, 28 September 2003, available at: http://nssdc.gsfc.nasa.gov/planetary/text/smart1_pr $20030928 . \mathrm{txt}$

Fassò, A., A. Ferrari, P.R. Sala, "Electron-photon transport in FLUKA: Status "Proceedings of the Monte Carlo 2000 Conference, Lisbon, October 23262000 , A. Kling, F. Barao, M. Nakagawa, L. T'avora, P. Vaz eds., Springer-Verlag, Berlin, p. 159-164 (2001).

Fassò, A., A. Ferrari, J. Ranft, P.R. Sala, "FLUKA: Status and Prospective for Hadronic Applications"Proceedings of the Monte Carlo 2000 Conference, Lisbon, October 23-26 2000, A. Kling, F. Bar ao, M. Nakagawa, L. T'avora, P. Vaz eds., Springer-Verlag, Berlin, p. 955-960 (2001).

Fassò, A., A. Ferrari, J. Ranft, P. R. Sala Fluka: a multiparticle transport code, CERN-2005-10(2005), INFN/TC 05/11, SLAC-R-773 


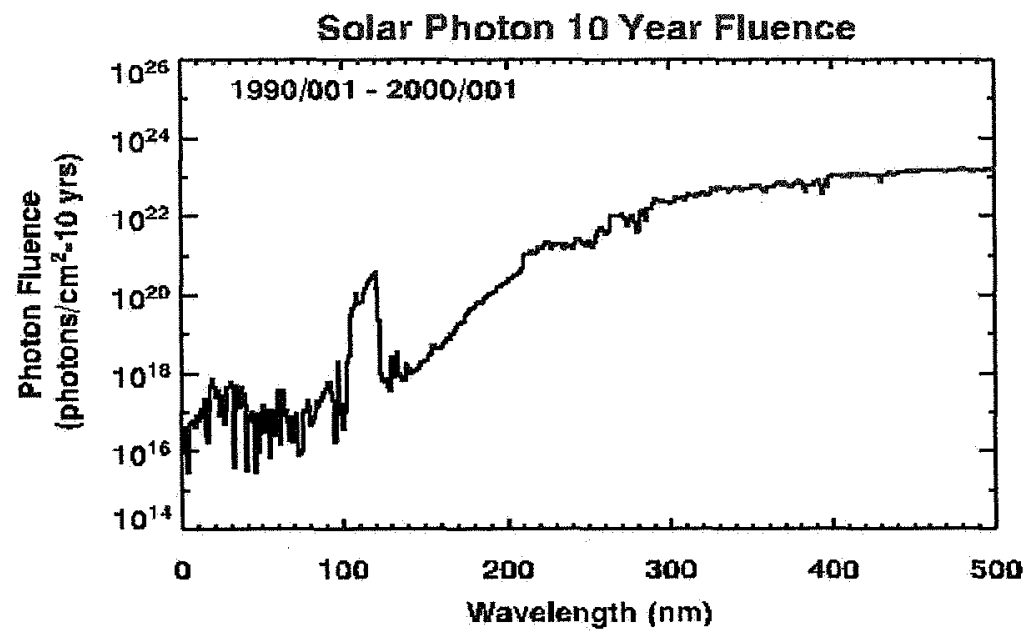

Figure 11. Solar UV/EUV 10 Year Photon Fluence. Photon fluence for the ten year period from 1 January 1990 to 1 January 2000 is obtained from integrating daily average spectra provided by the SOLAR2000 model.

Fassò, A., A. Ferrari, S. Roesler, P. R. Sala, G. Battistoni, F. Gadioli, M. V. Garzelli, F. Ballarini, A. Ottolenghi, A. Empl, J. Ranft, "The Physics Models of FLUKA: status and recent developments" Computing in High Energy and Nuclear Physics 2003 Conference (DHEP2003), La Jolla, CA, USE, March 24-28 2003, (paper MOMT005), eConf C0303241 (2003), arXiv:hep-ph/0306267

Feldman W.C., J.R. Asbridge, S.J. Bame, and J.T. Gosling, Plasma and Magnetic Fields from the Sun, in The Solar Output and its Variation, (ed.) Oran R. White, Colorado Associated University Press, Boulder, 1977.

Fennell, J.F., H.C. Koons, M.W. Chen, and J.B. Blake, Internal charging, a preliminary environmental specification for satellites, IEEE Trans. Plasma Sci., 28, 2029-2036, 2000.

Fillius, W., and I. Axford, Large scale solar modulation of $\leq 500 \mathrm{MeV} / \mathrm{Nucleon}$ galactic cosmic rays seen from 1 to $30 \mathrm{AU}, J$. Geophys. Res., 90, 517-520, 1985.

Freeman J.W. et al. (1973) JGR, 78, 4560-4567.

Freeman, J.W., and M. Ibrahim, Lunar electric fields, surface potential, and associated plasma sheaths, Earth, Moon, and Planets, 14, 103-114, 2004.

Garrett, H.B., and A. Hoffman, Comparison of spacecraft charging environments at the Earth, Jupiter, and Saturn, $6^{\text {th }}$ Spacecraft Charging Technology conference, AFRL-VS-TR-20001578, September 2000.

Halekas, J.S., S.D. Bale, D.L. Mitchell, and R.P. Lin, Electron and magnetic fields in the lunar plasma wake, J. Geophys. Res., 110, A07222, doi:10.1029/2004J A010991, 2005.
Halekas, J.S., Mitchell, D.L., Lin, R.P., et al. Evidence for negative charging of the lunar surface in shadow, Geophys. Res. Lett., 29(10), 1435, doi:10.1029/2001GL014428, 2002.

Howe, Jr., H.C., and J.H. Biinsack, Explorer 33 and 35 plasma observations of magnetosheath flow, $J$. Geophys. Res., 77, 3334 -3344, 1972.

Knott, K., Electrostatic charging of the lunar surface and possible consequences, J. Geophys. Res., 78, 3172-3175, 1973.

Koons, H. C., J. E. Mazur, R. S. Selesnick, J. B. Blake, J. F. Fennell, J. L. Roeder, and P. C. Anderson, The impact of the space environment on space systems, $6^{\text {th }}$ Spacecraft Charging Technology Conference, Air Force Research Laboratory, Hanscom AFB, MA, 26-29 October, 1998.

Lindeman, R., J. W. Freeman, and R. R. Vondrak, Ions from the lunar atmosphere, Proc. Lunar Science Conf., 4, 2889-2896, 1973.

Lozier, D., K. Galal, D. Folta, and M. Beckman, Lunar Prospector mission design and trajectory support, AAS Paper 98-323, 1998.

Mall, U.A., and N. Borisov, Electric potential distribution on the nightside of the Moon, Lunar and Planetary Science XXXII, Paper 1538, 2001.

Manka, R.H., and F.C. Michel, Lunar ion energy spectra and surface potential, Geochim. Cosmochim. Acta, 3, 2897, 1973 .

Meeus, J., Mathematical Astronomy Morsels, Willmann-Bell, Inc., Richmond, Virginia, 1997.

Minow, J.I., R.L. Altstatt, and L.F. Neergaard, Interplanetary Radiation and Internal Charging Environment Models for Solar Sails, presented at the 
Solar Sail Technology and Applications Conference, Greenbelt, MD, 28-29 September 2004a.

Minow, J.I., W.C.Blackwell,Jr., and A. Diekmann, Plasma Environment and Models for L2, AIAA Paper 2004-1079, 42nd AIAA Aerospace Sciences Meeting and Exhibit, Reno, NV, 5-8 January 2004b.

Minow, J.I., W.C. Blackwell, L.F Neergaard, S.W Evans, D.M. Hardage, and J.K. Owens, Charged Particle Environment Definition for NGST: L2 Plasma Environment Statistics, Proc. SPIE, 4013, 942-953, UV, Optical, and IR Space Telescopes and Instruments VI, J.B. Breckinridge and P. Jakobsen, eds., 2000

Minow, J.I., L.N. Parker, and R.L. Altstatt, Radiation and internal charging environments for thin dielectrics in interplanetary space, presented at the $9^{\text {th }}$ Spacecraft Charging Technology Conference, Tsukuba, Japan, 4-8 April 2005.

Minow, J.I., L.N. Parker, R. Altstatt, and W. Skipworth, Ion Flux Environments in Interplanetary Space, AIAA-2006-0473, 44 ${ }^{\text {th }}$ AIAA Aerospace Sciences Meeting, Reno, NV, January 2006.

NASA 4002 , Avoiding Problems Caused by Spacecraft On-Orbit Internal Charging Effects, NASA-HDBK4002, 17 February 1999.

McKay, D. S., J. L. Carter, W. W. Boles, C. C. Allen, and J.H. Alton, JSC-1: A new lunar soil simulant, in Engineering, Construction, and Operations in Space IV, pp. 857- 866, Am. Soc. of Civ. Eng., Reston, Va., 1994.

Ogilvie, K. W., J. T. Steinberg, R. J. Fitzenreiter, C. J. Owen, A. J. Lazarus, W. M. Farrell, and R. B. Torbert, Observations of the lunar plasma wake from the WIND spacecraft on December 27, 1994., Geophys. Res. Lett., 23, 1255- 1258, 1996.

Olsen, R.C., Record charging events from Applied Technology Satellite 6, J. Spacecraft and Rockets, 24, 362 - 366, 1986.

Orloff, R.W., Apollo by the Numbers: A Statistical Reference, NASA SP-2000-4029, NASA Headquarters, Washington, DC, 2000.

Parker, E.N., The passage of energetic particles through interplanetary space, Planet. Space Sci., 13, 9-49, 1965.

Reasoner, D. L., Burke, W. J.: Direct observations of the lunar photoelectron layer. Geochim. Cosmochim. Acta Suppl. 4, 2639, 1972.

Regeon, P.A., R.J. Chapman, and R. Baugh, Clementine, "The deep space program science experiment," Paper IAA-L-0501, presented at the International Academy of Astronautics (IAA) International Conference On Low-Cost Planetary Missions, Johns Hopkins University, Applied Physics Laboratory, Laurel, Maryland, 12-15 April 1994.
Sawyer, D. and J. Vette, AP-8 trapped proton environment for solar maximum and solar minimum, National Space Science Data Center, Report 76-06, Greenbelt, Maryland, 1976.

Seltzer, S. M., SHIELDOSE, A Computer Code for Space-Shielding Radiation Dose Calculations, National Bureau of Standards, NBS Technical Note 1116, U.S. Government Printing Office, Washington, D.C., 1980.

Seltzer, S. M., Updated calculations for routine spaceshielding radiation dose estimates: SHIELDOSE-2, NIST Publication NISTIR 5477, Gaithersburg, MD., December 1994.

SSP-30512, Space Station Ionizing Radiation Design Environment, SSP 30512, Revision C., 3 June 1994.

Tobiska, W.K., "SOLAR2000 irradiances for climate change, aeronomy, and space system engineering," Adv. Space Res., 34, 1736-1746, 2004.

Tobiska, W.K, T. Woods, F. Eparvier, R. Viereck, L. Floyd, D. Bouwer, G. Rottman, and O.R. White, "The SOLAR2000 empirical solar irradiance model and forecast tool," J. Atm. Solar Terr. Phys., 62, $1233-1250,2000$.

Tylka, A.J., J.H. Adams, Jr., P.R. Boberg, B. Brownstein, W.F. Dietrich, E.O. Flueckiger, E.L. Petersen, M.A. Shea, D.F. Smart, and E.C. Smith, CREME96: A Revision of the Cosmic Ray Effects on Micro-Electronics Code, IEEE Trans. Nuclear Sci., 44, 2150-2160, 1997.

Vette, J., The AE-8 trapped electron model environment, National Space Science Data Center, Report 91-24, Greenbelt, Maryland, 1991.

Vyshlov, A. S.: Preliminary results of circum-lunar plasma research by the Luna 22 spacecraft. Space Research 16, 945, 1974.

Vyshlov, A. S., Savich, N. A.: Observations of radio source occultations by the moon and the nature of the plasma near the moon. Kosmich-Issledovanija 16/4, 551-556, 1978.

Wilson, J.W., F.A. Cucinotta, H. Tai, L.C. Simonsen, J.L. Shinn, S. A. Thibeault, and M.Y. Kim, Galactic and solar cosmic ray shielding in deep space, NASA Technical Paper 3682, December 1997.

Xapsos, M.A., J.L. Barth, E.G. Stassinopoulos, E.A. Burke, and G.B. Gee, Space Environment Effects: Model for Emission of Solar Protons (ESP)Cumulative and Worst-Case Event Fluences, NASA-TP-1999-209763, December 1999. 


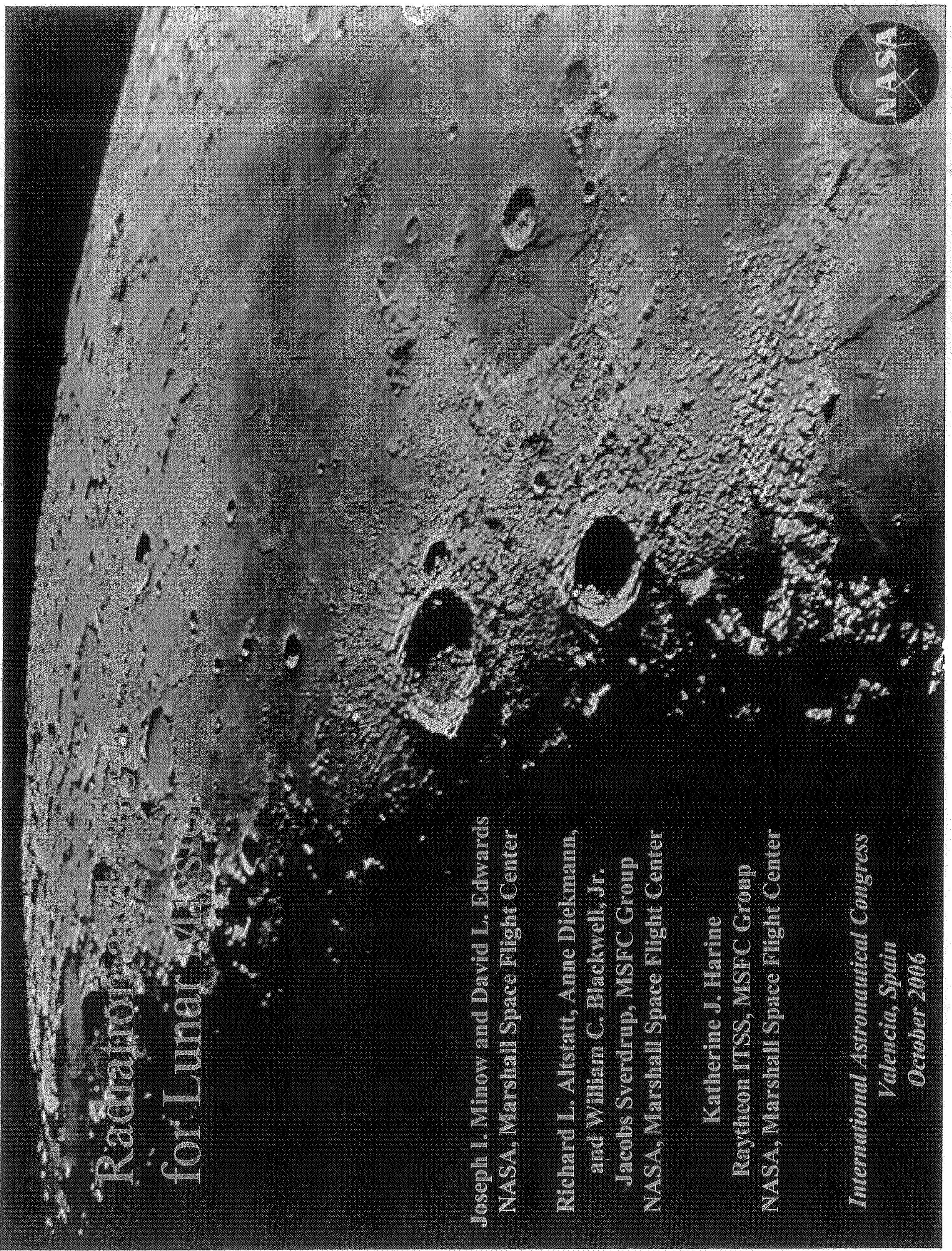




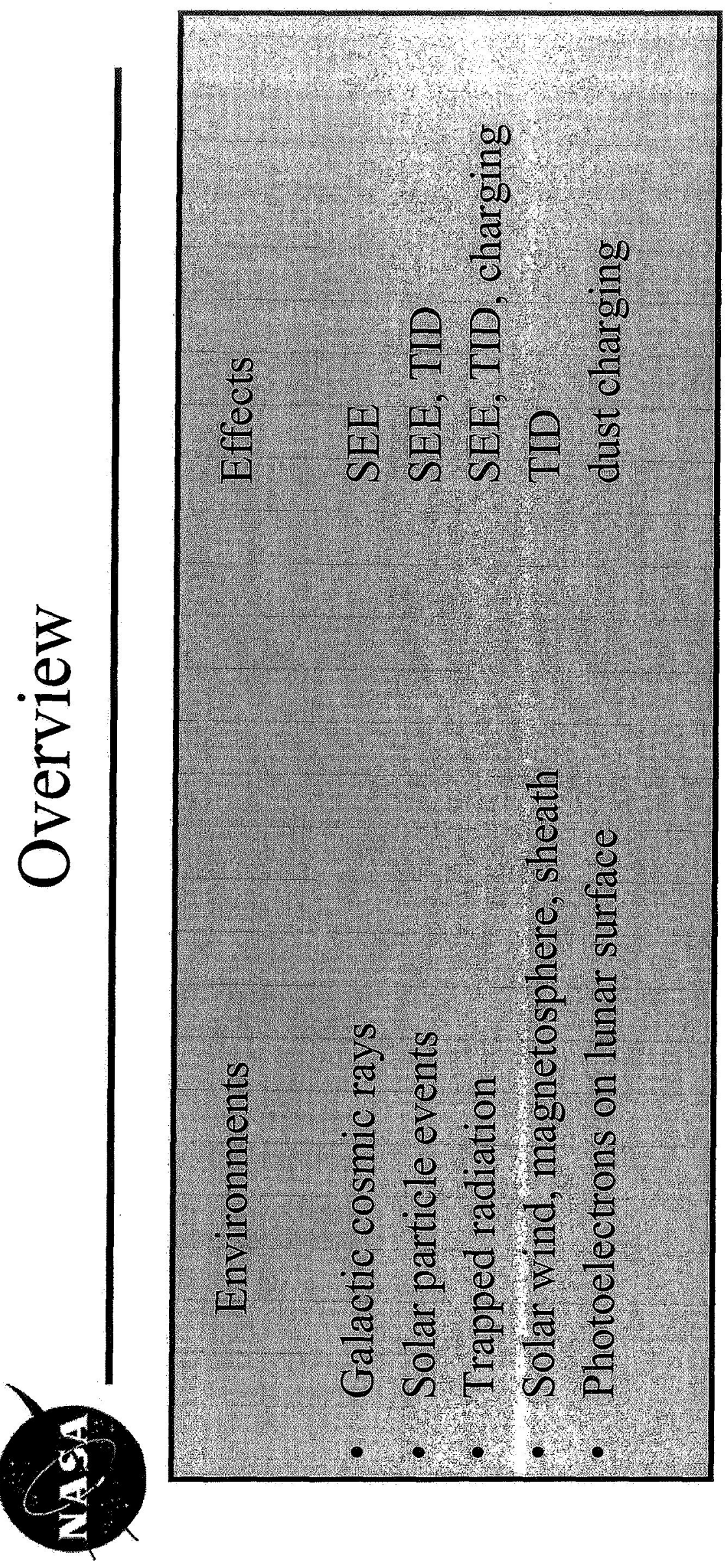

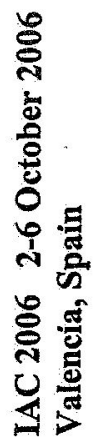




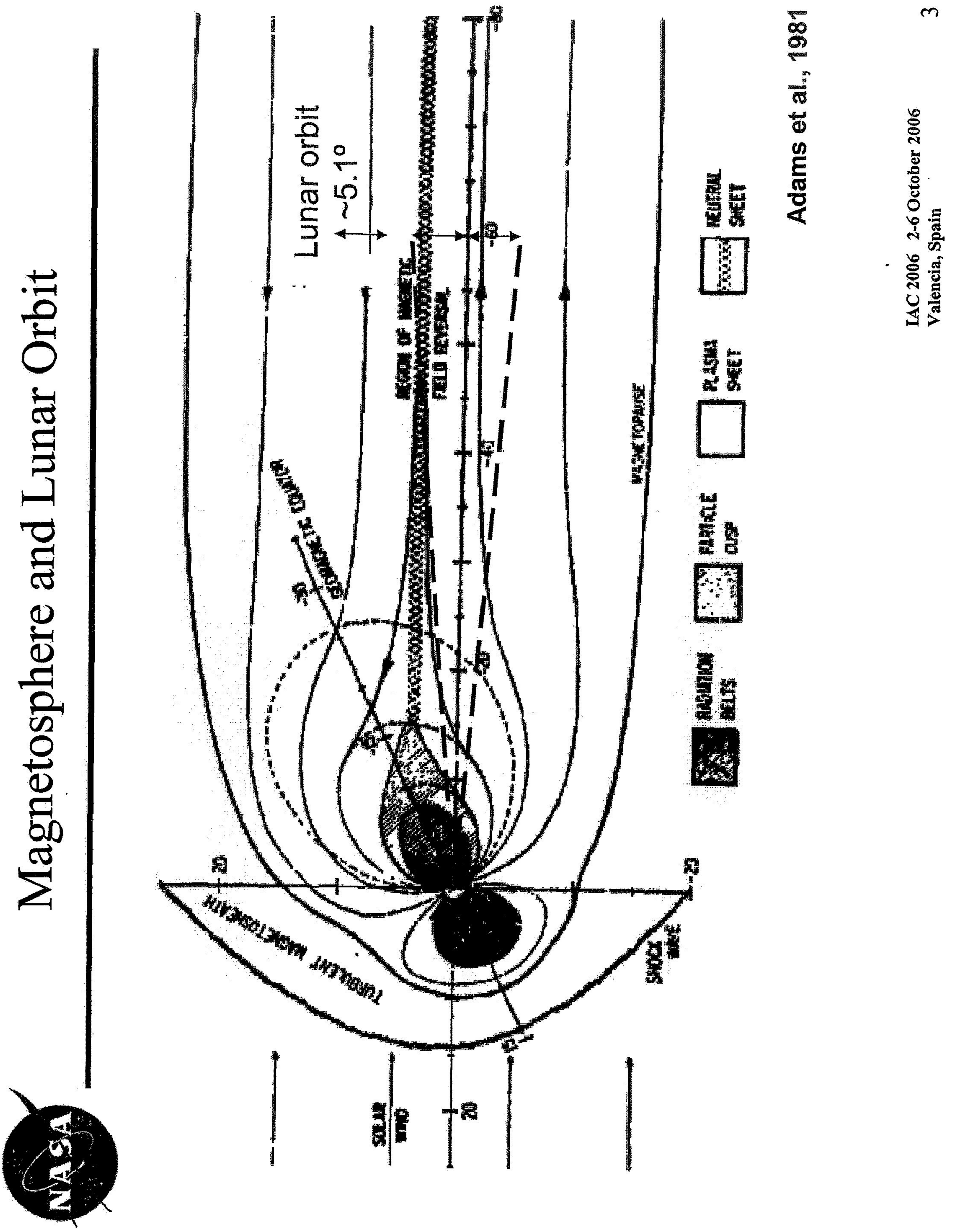




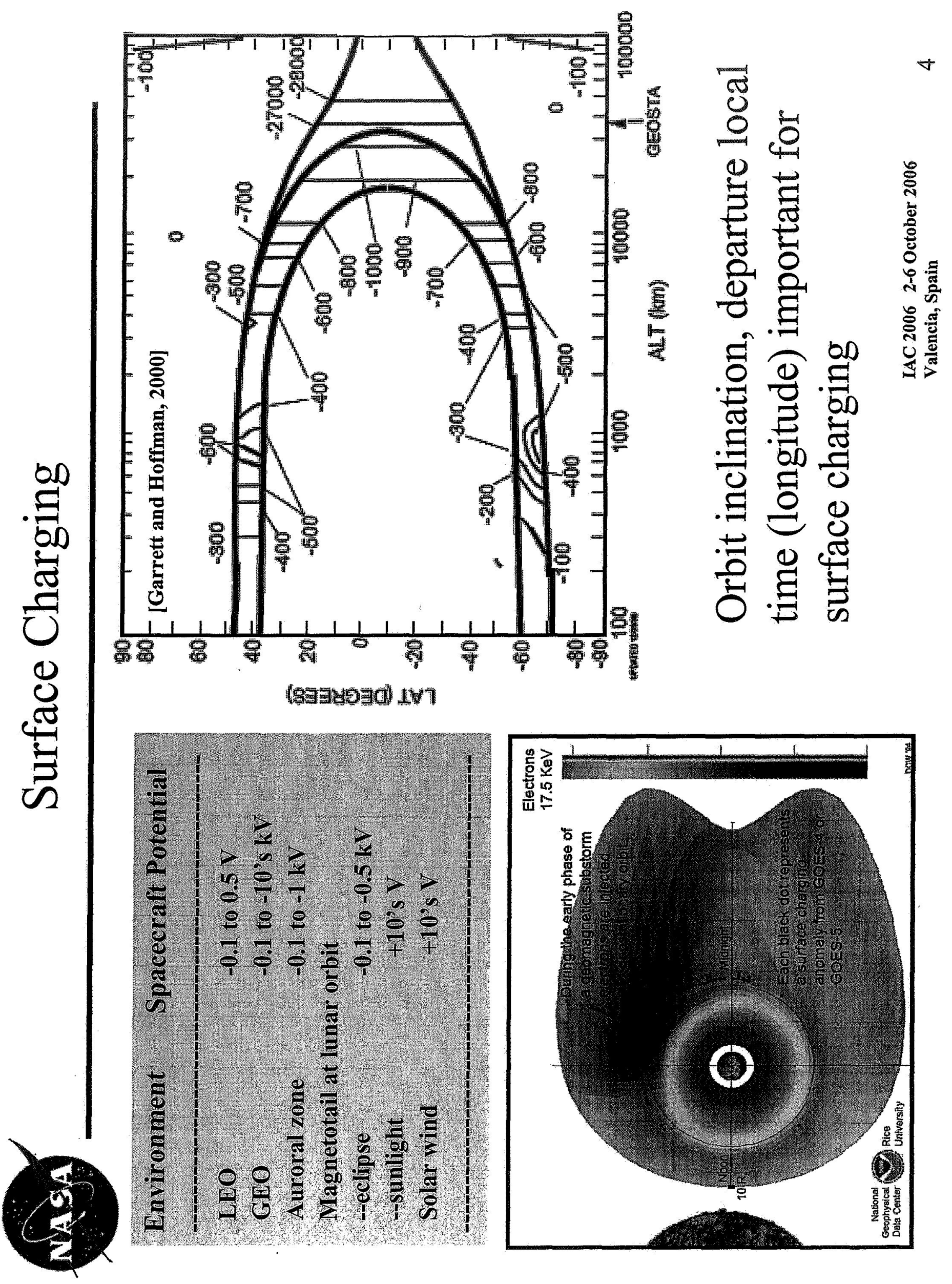



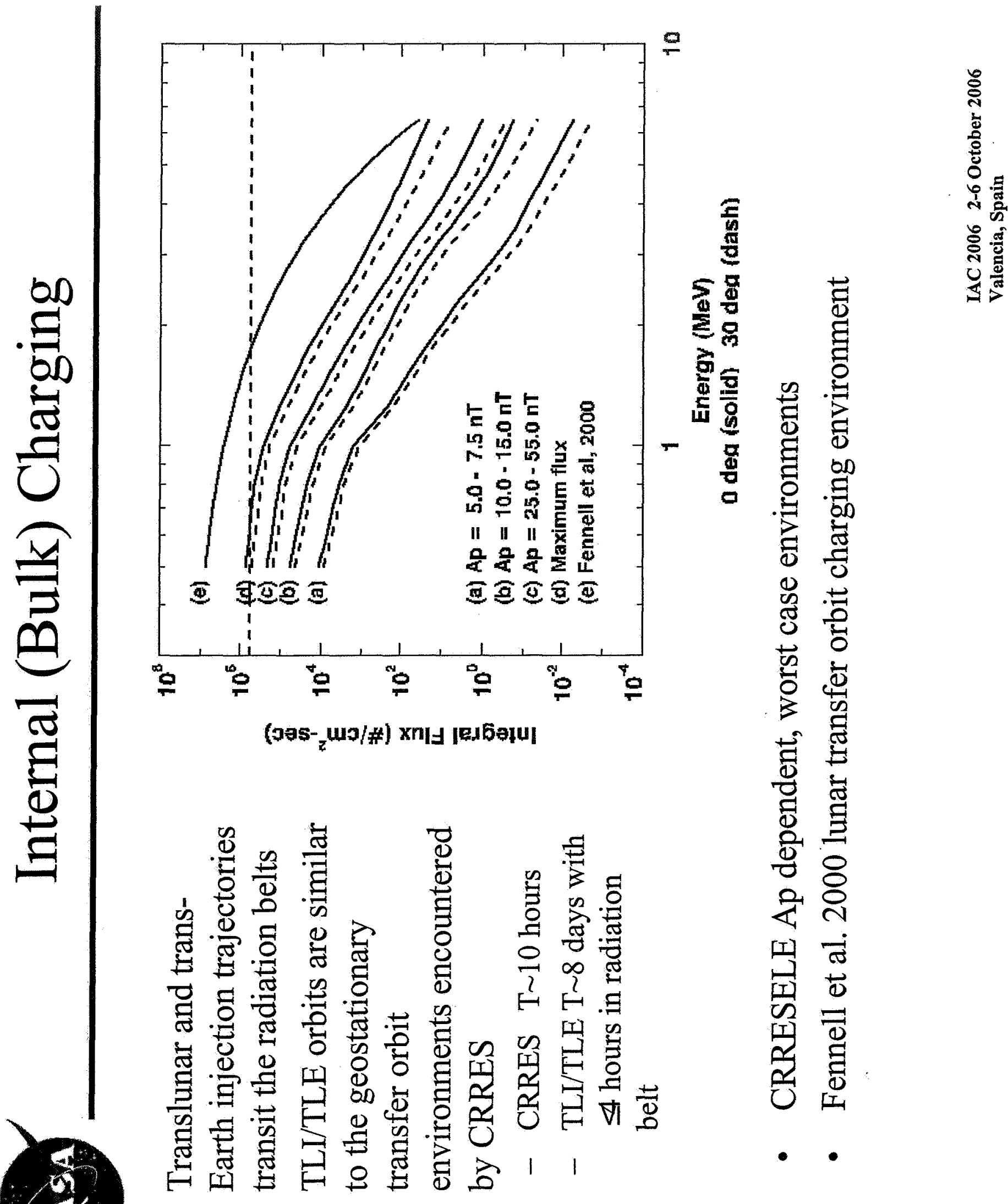

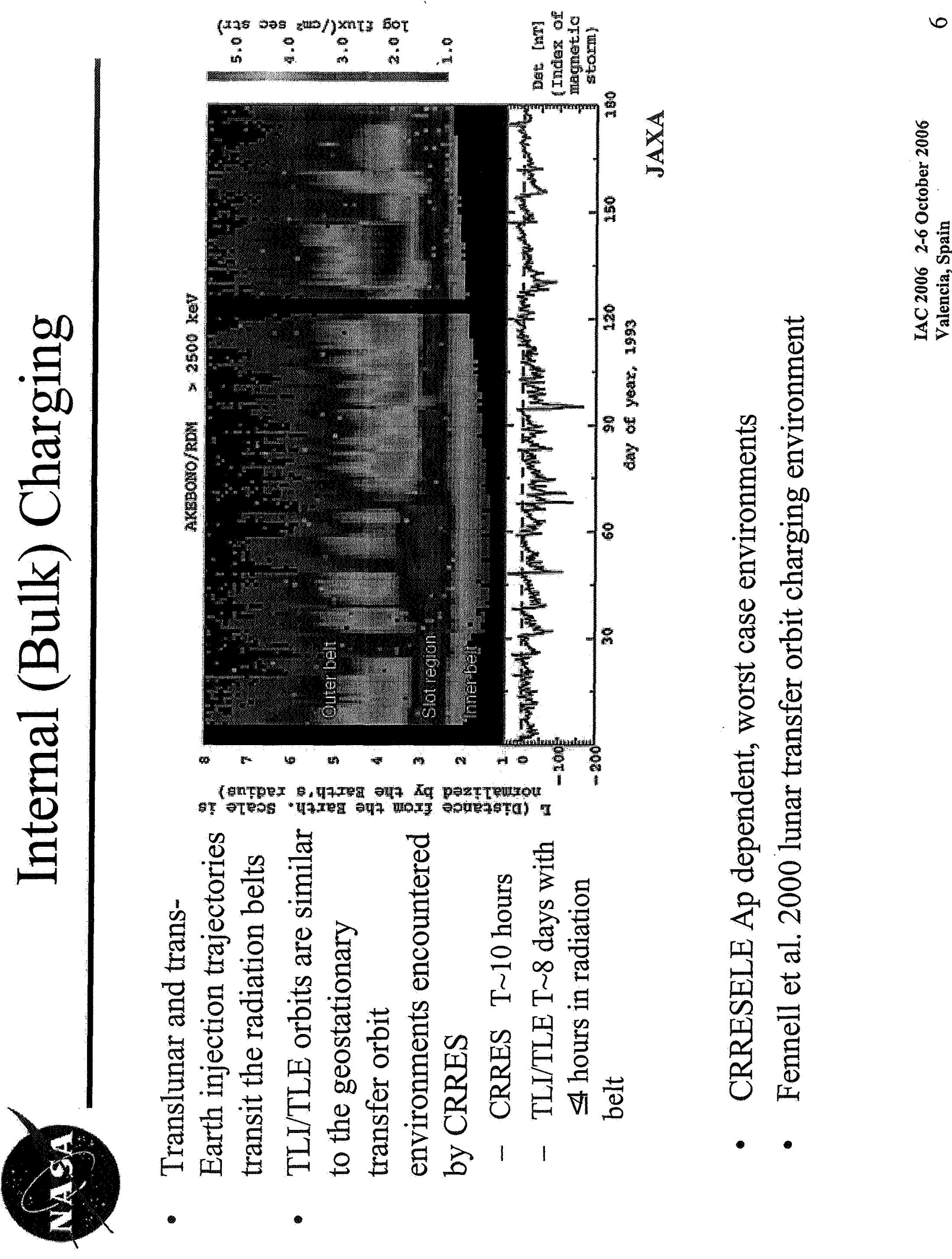


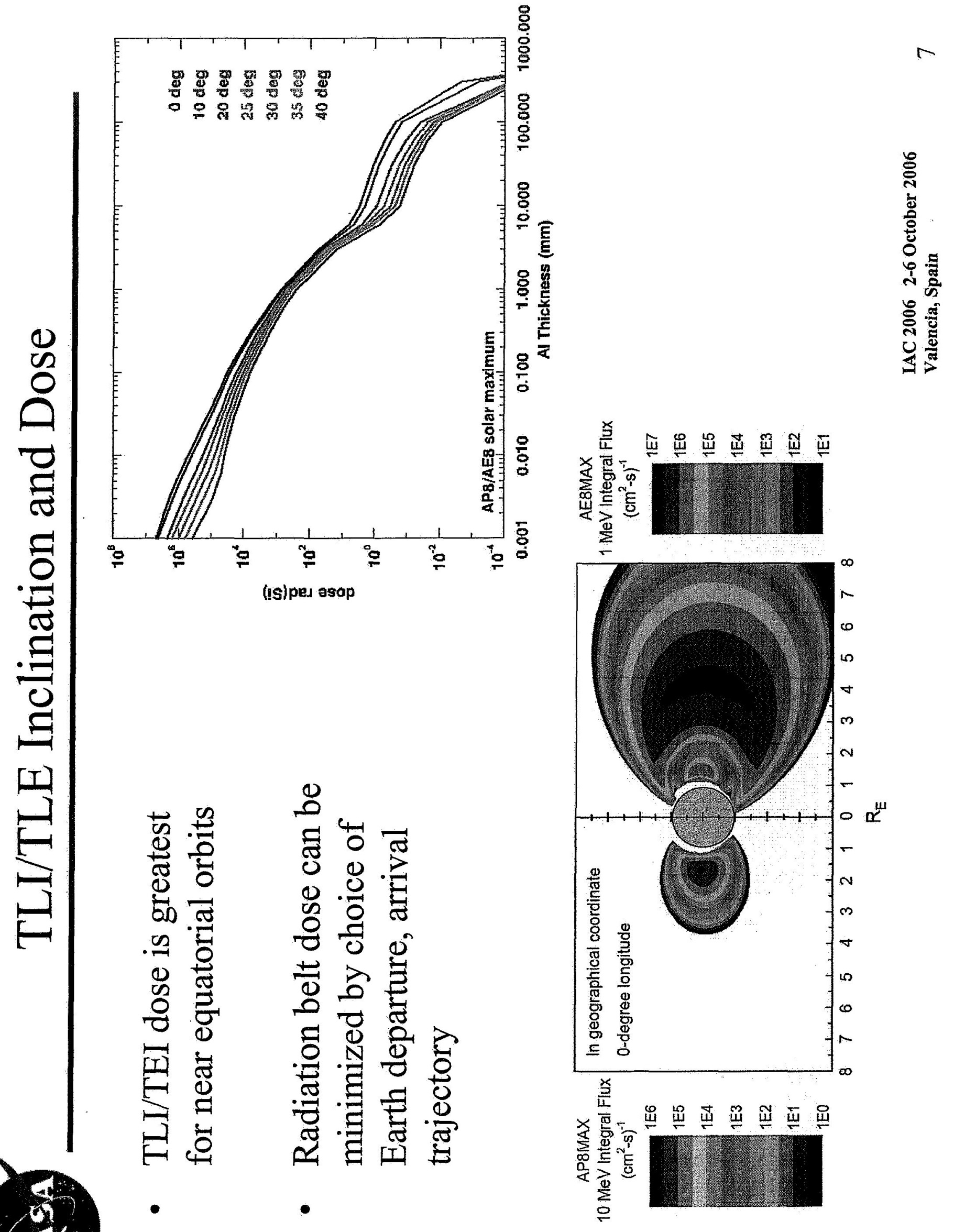




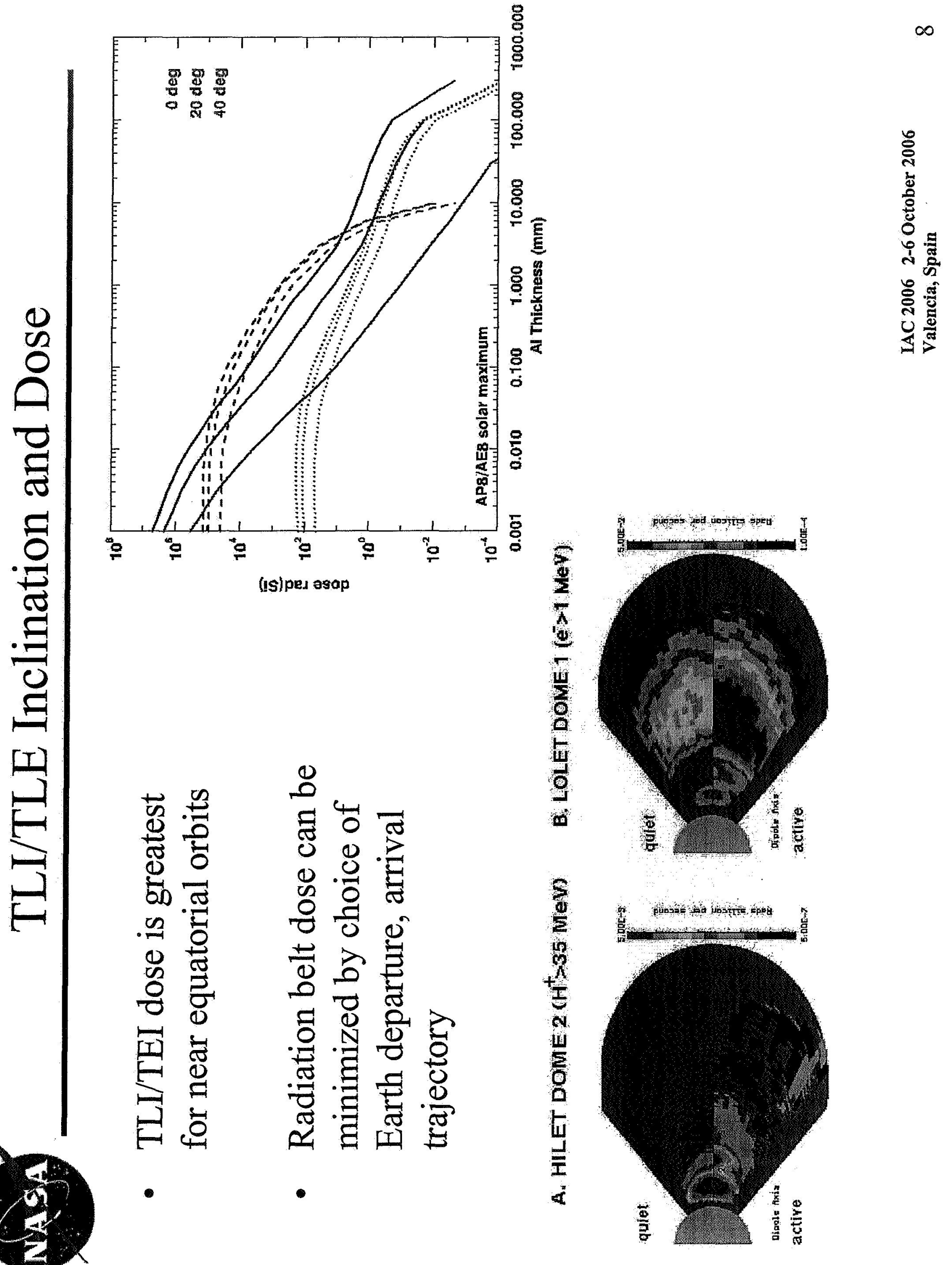




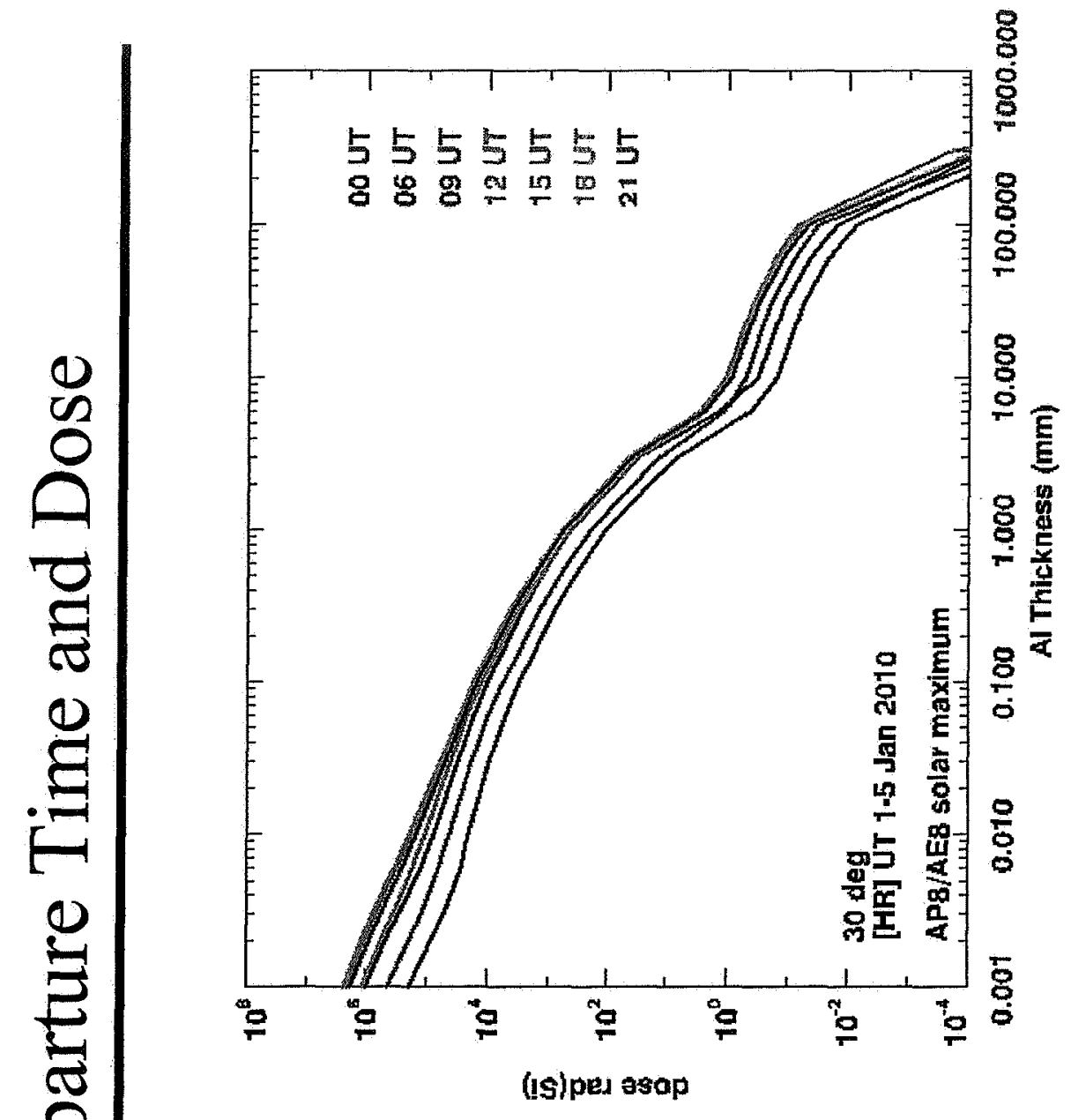

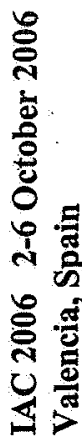

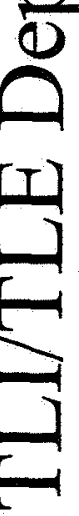

5

0
$\frac{8}{0}$
$\frac{8}{0}$

\&

팔
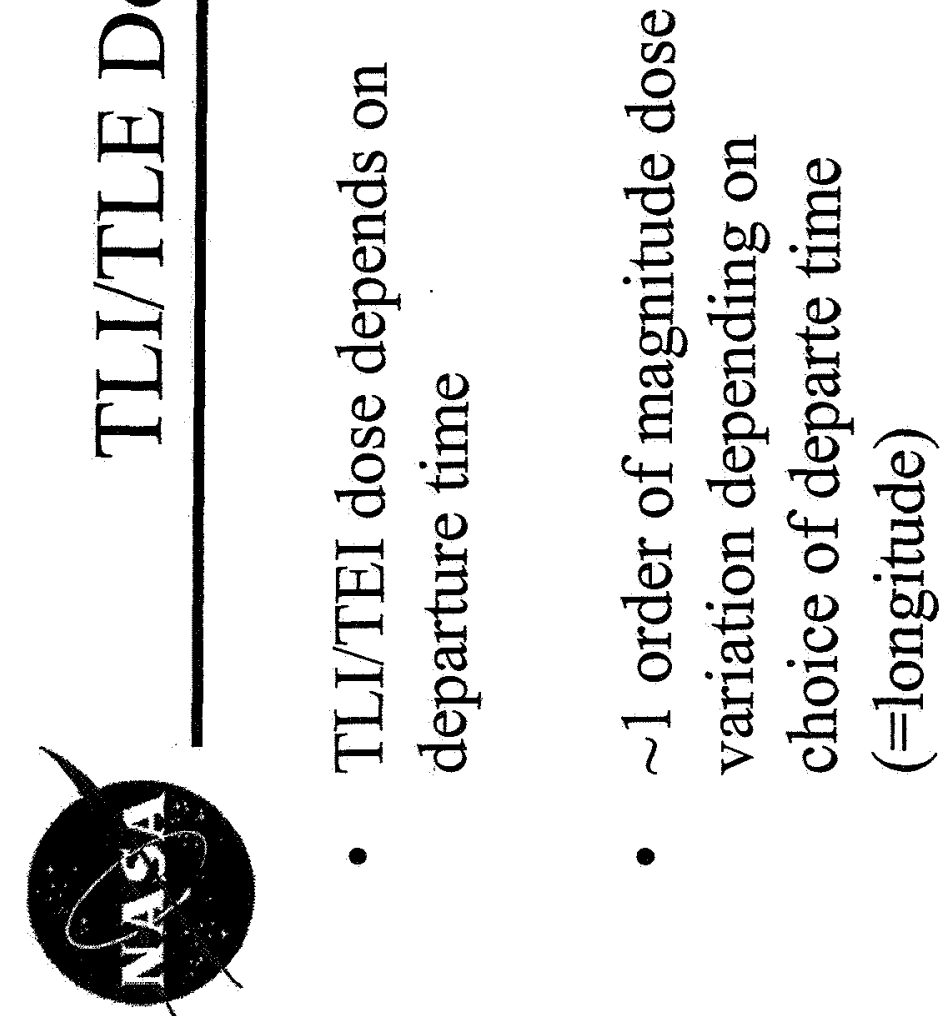

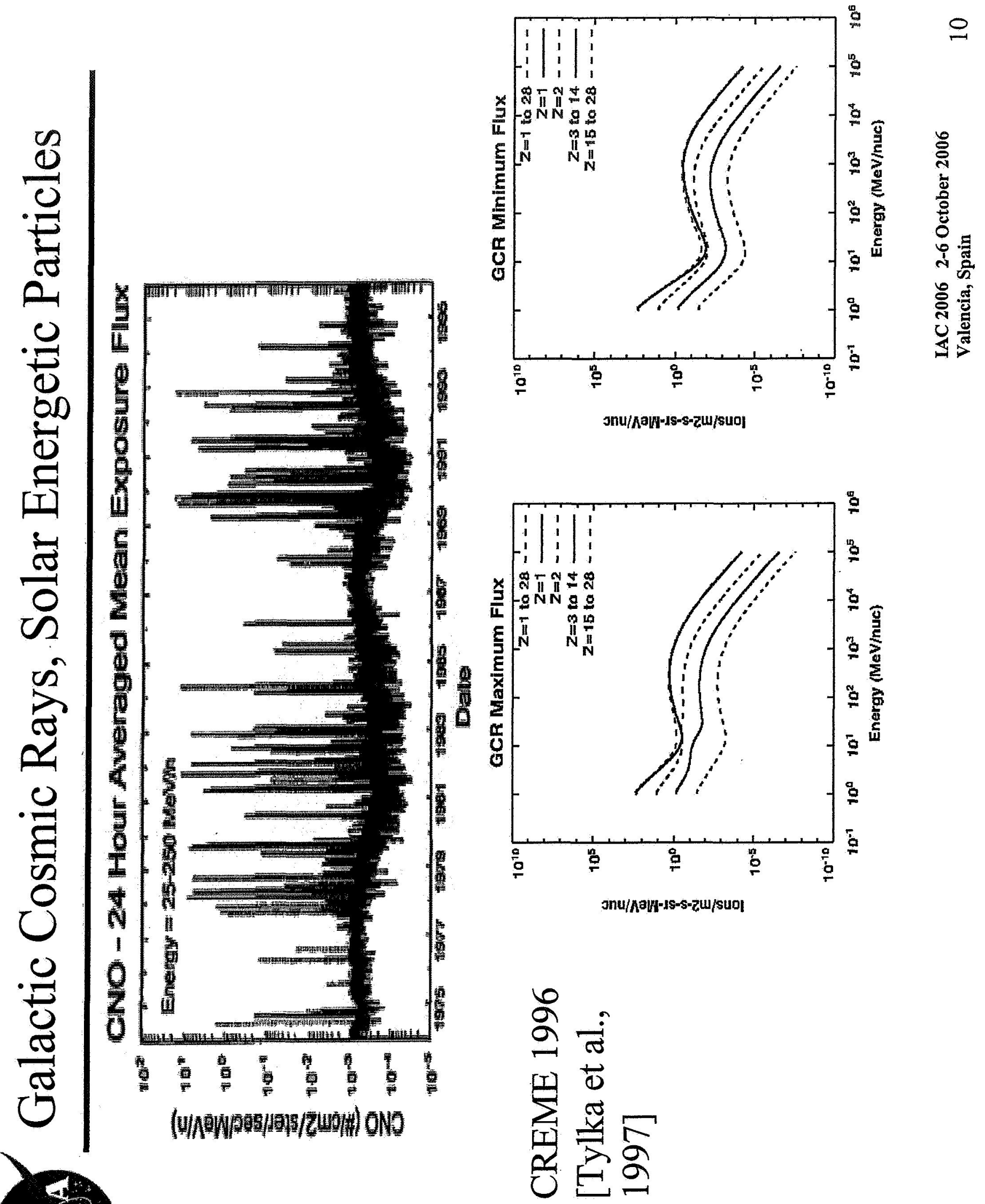

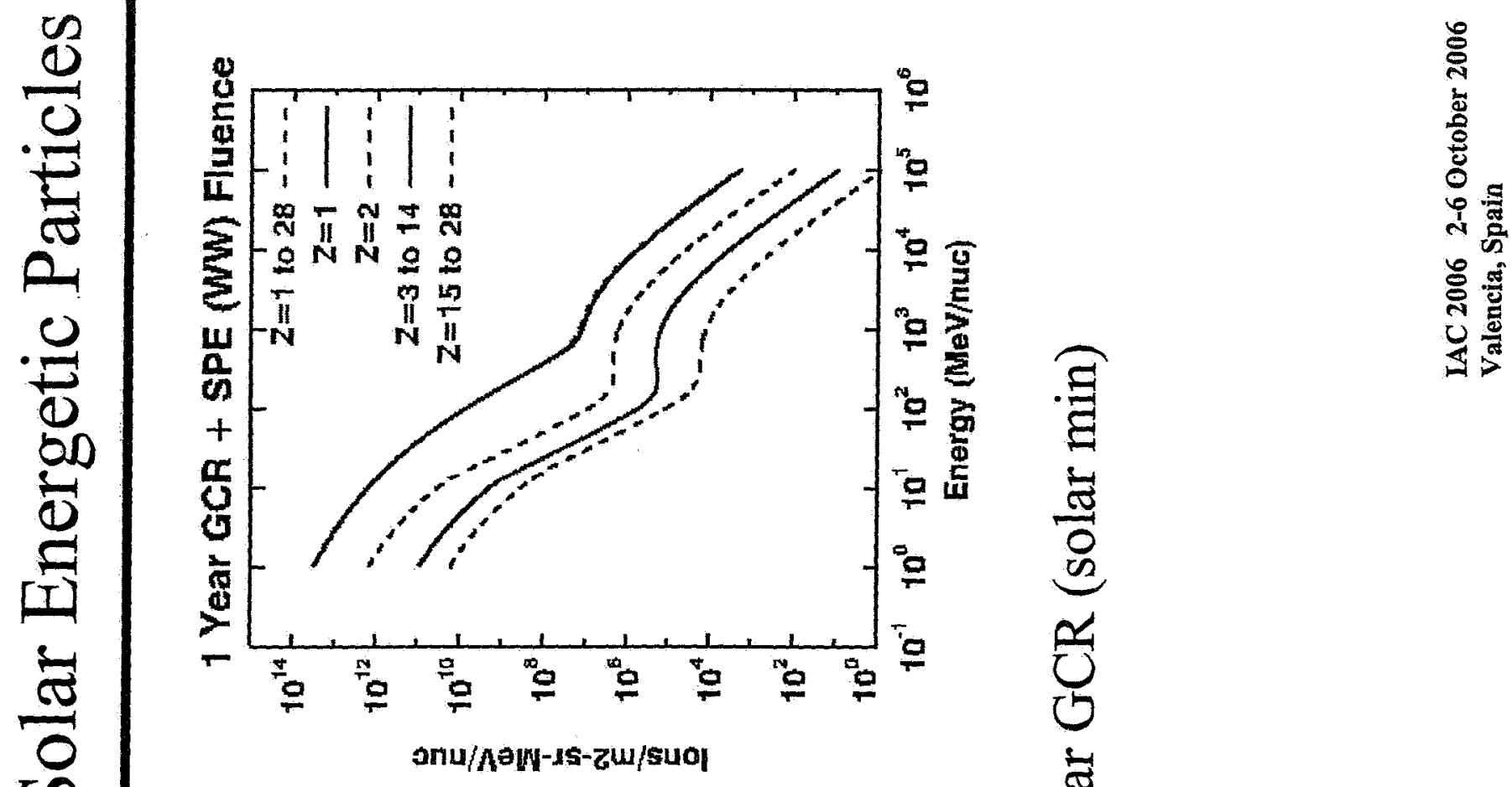

0
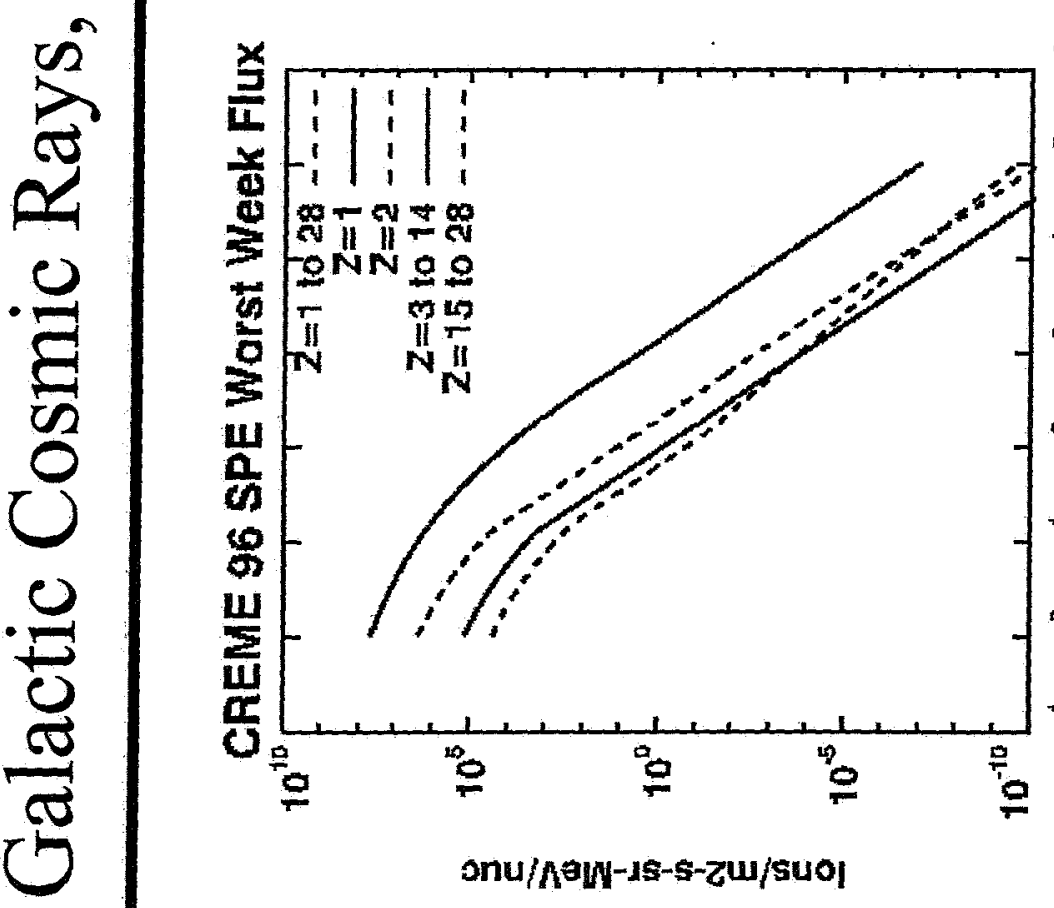

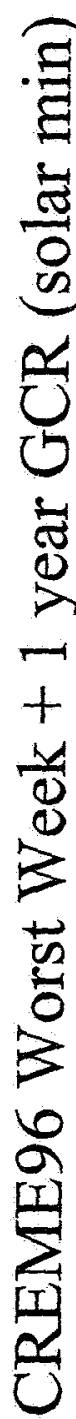

5

onu/helly-15-s-zw/suol 


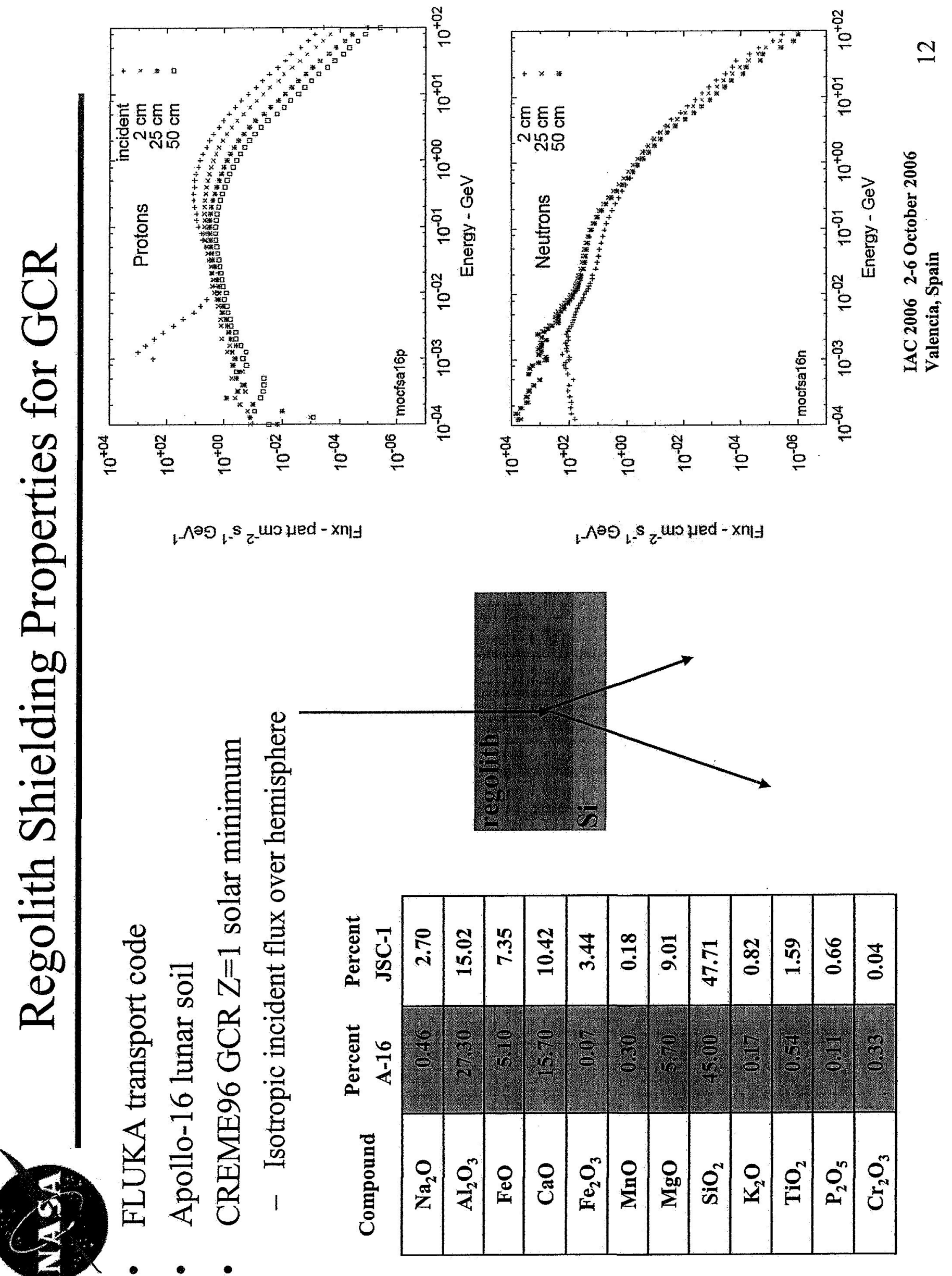




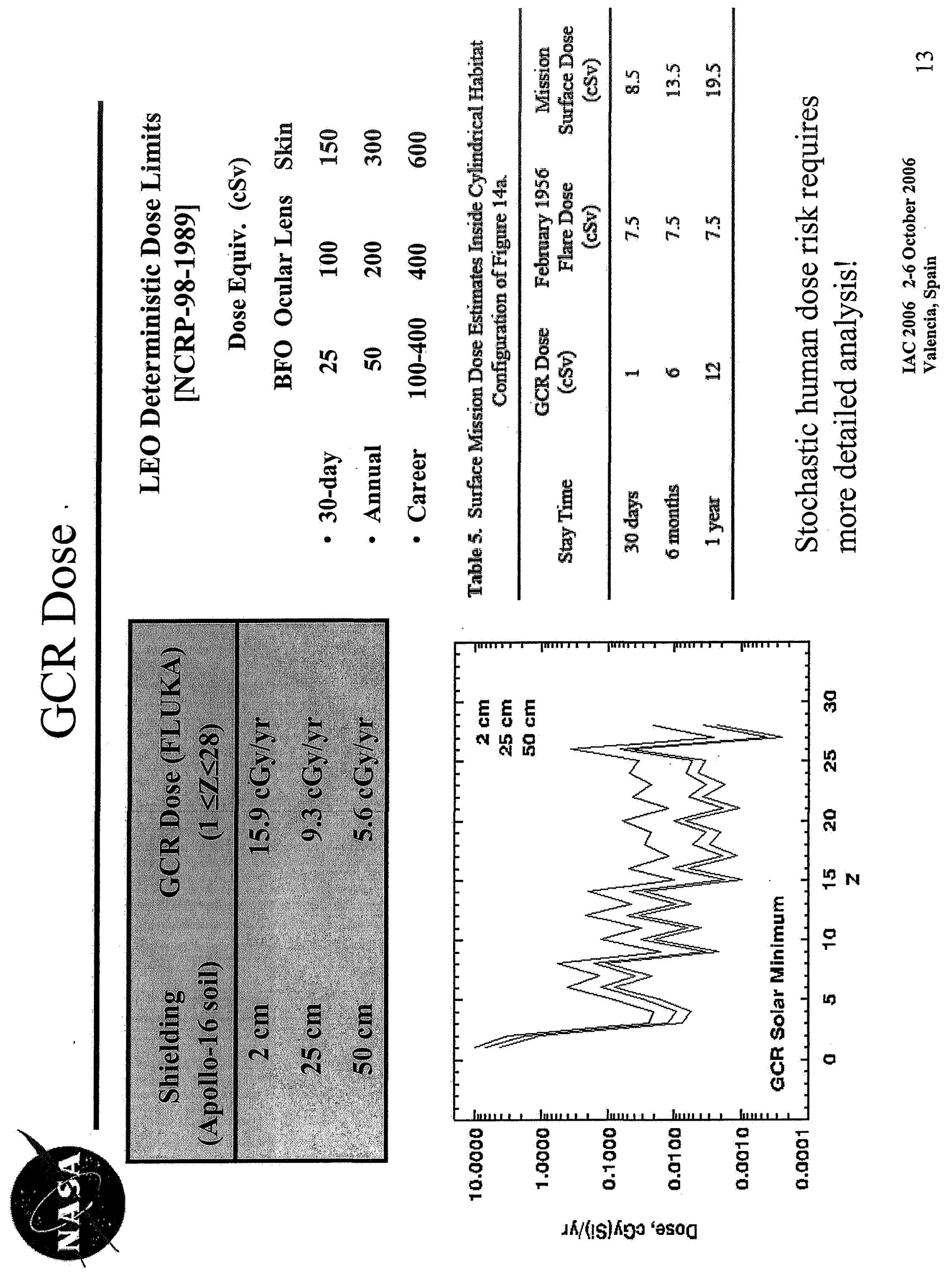



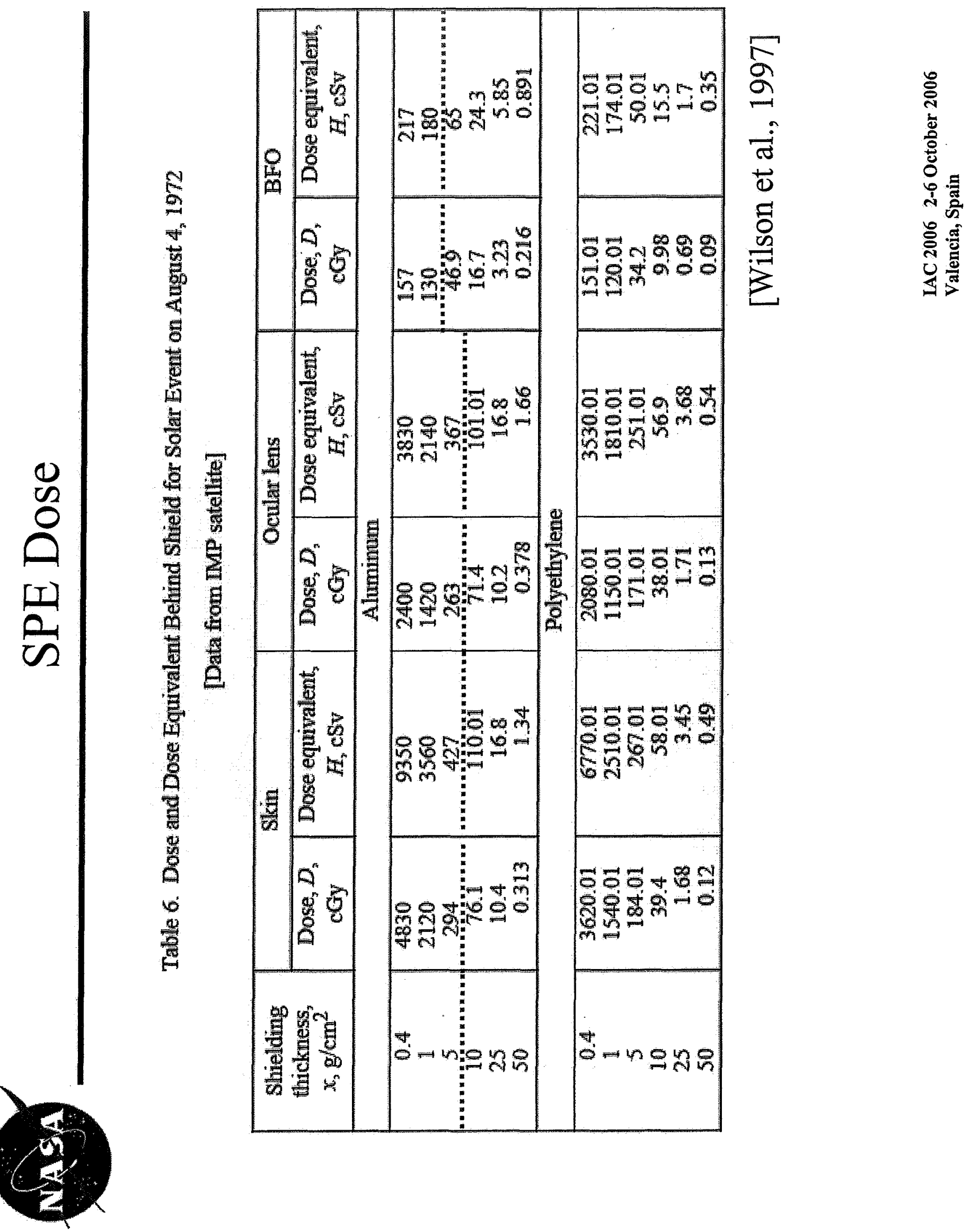

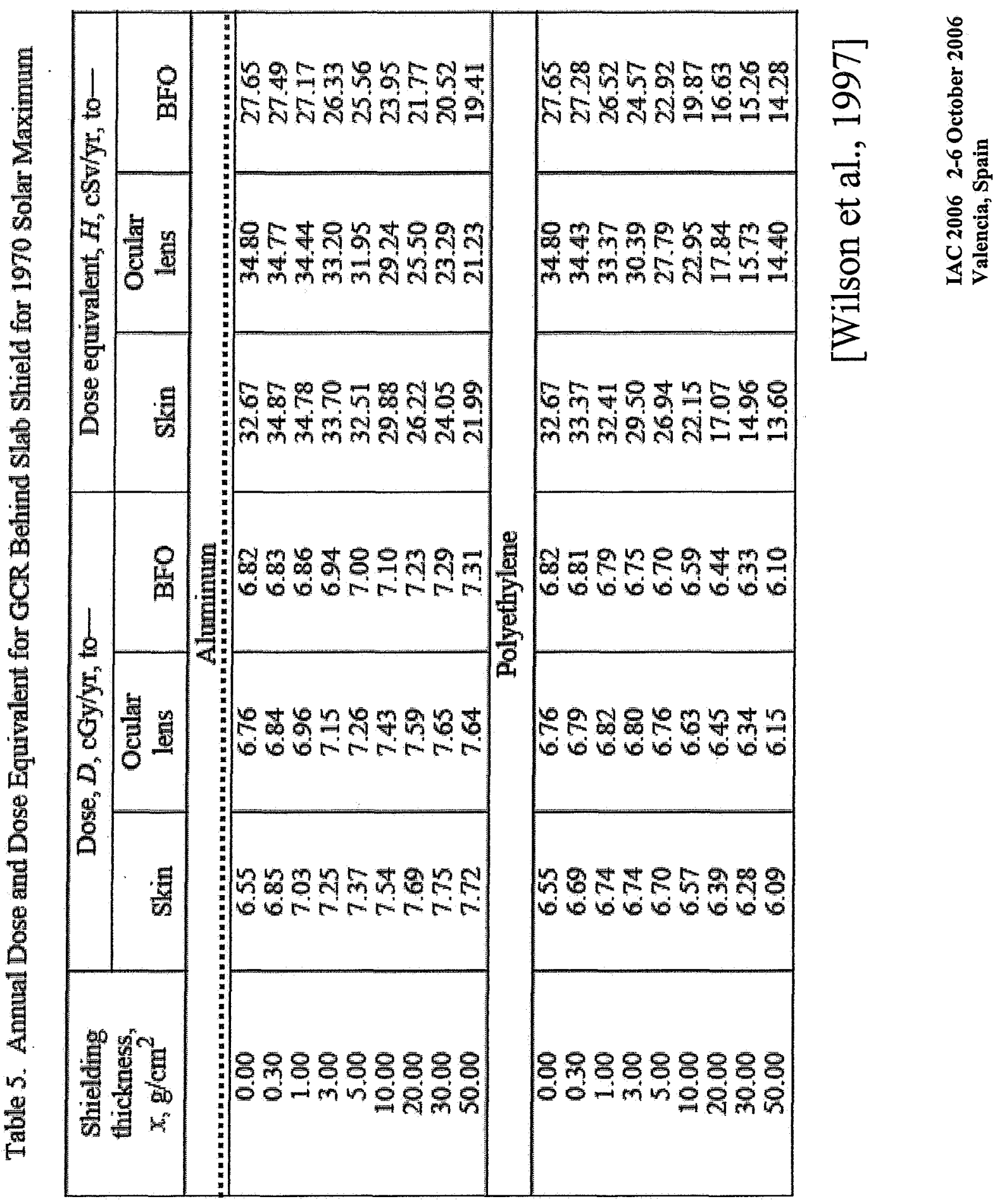


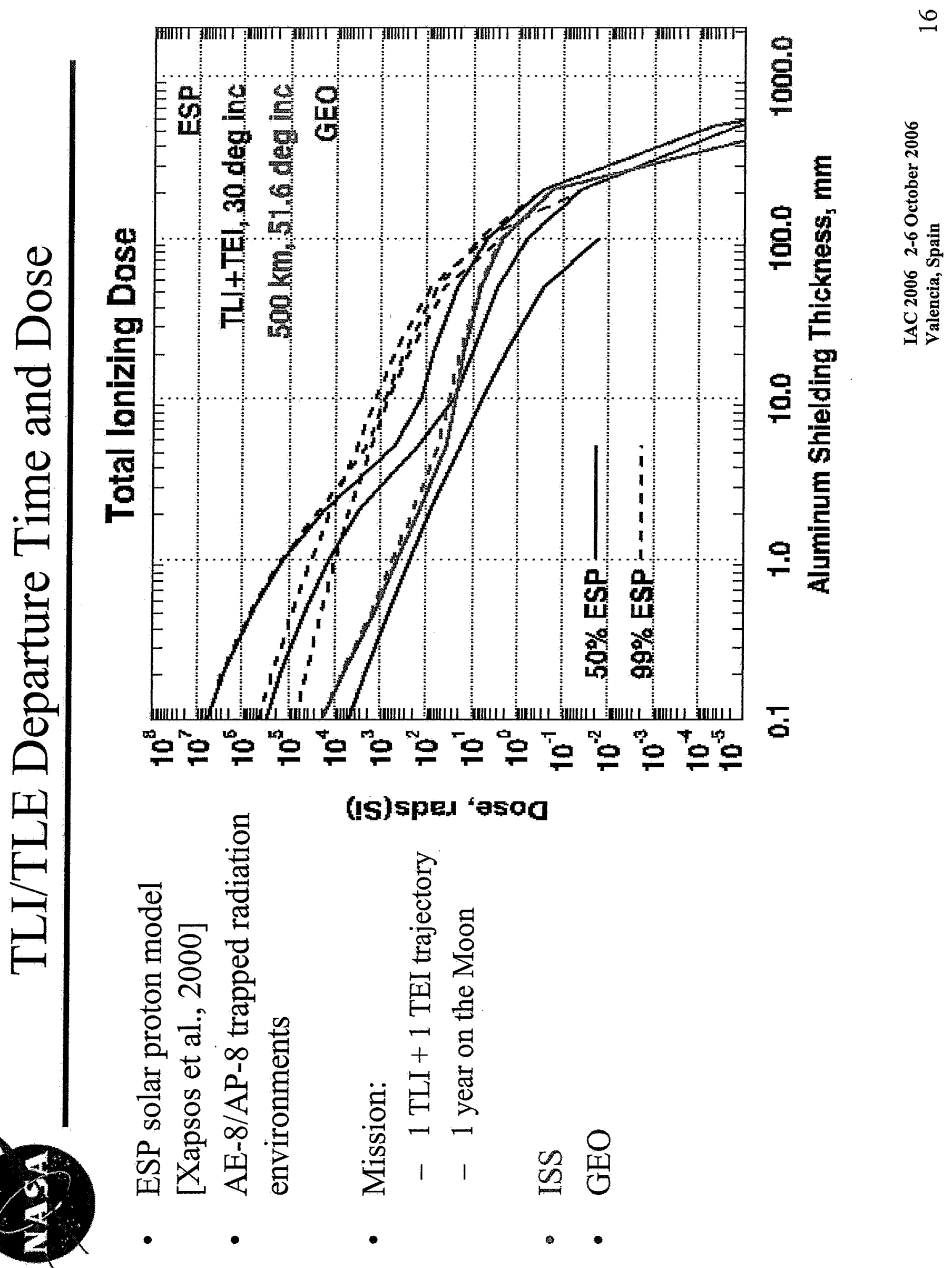




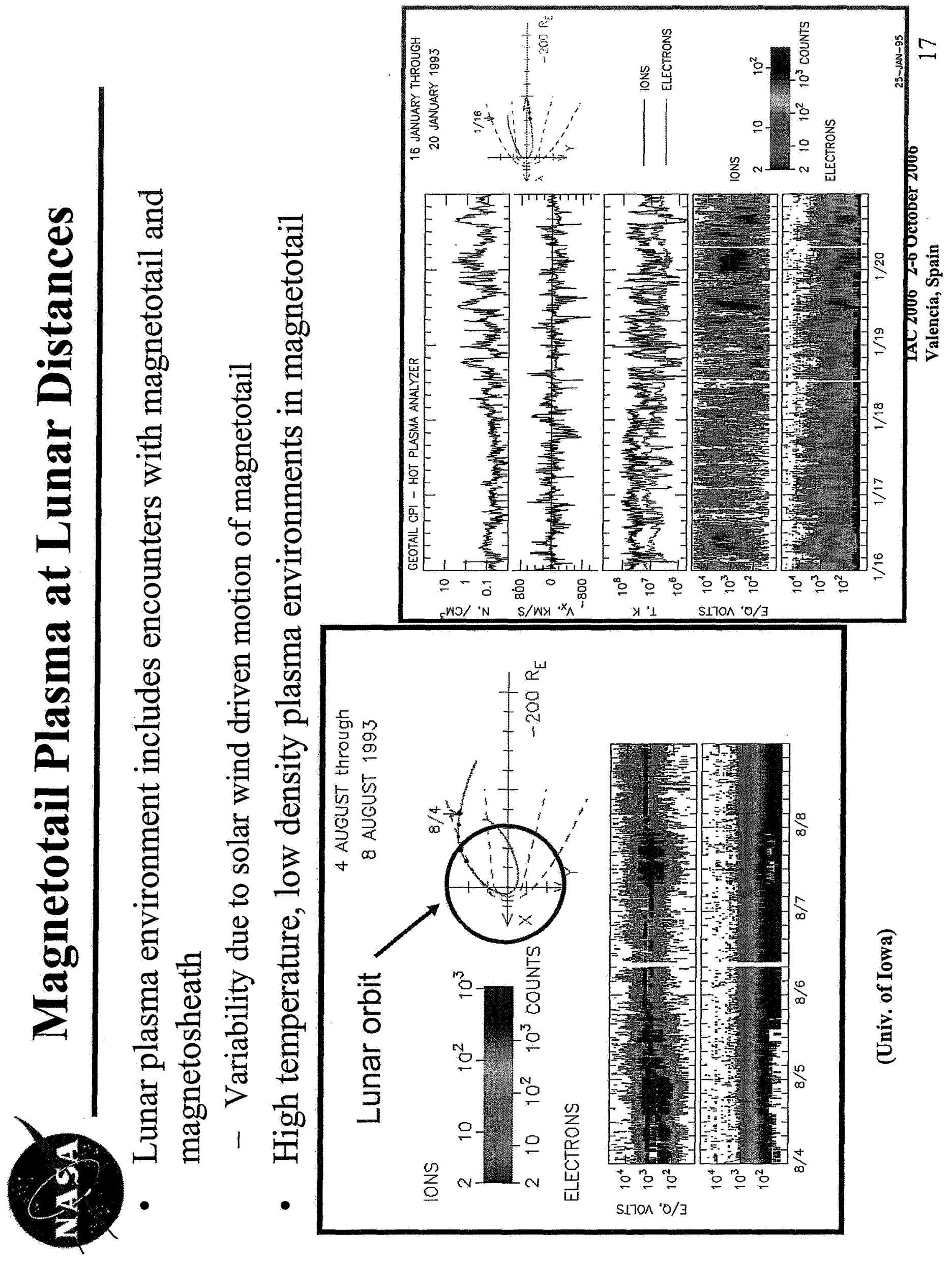




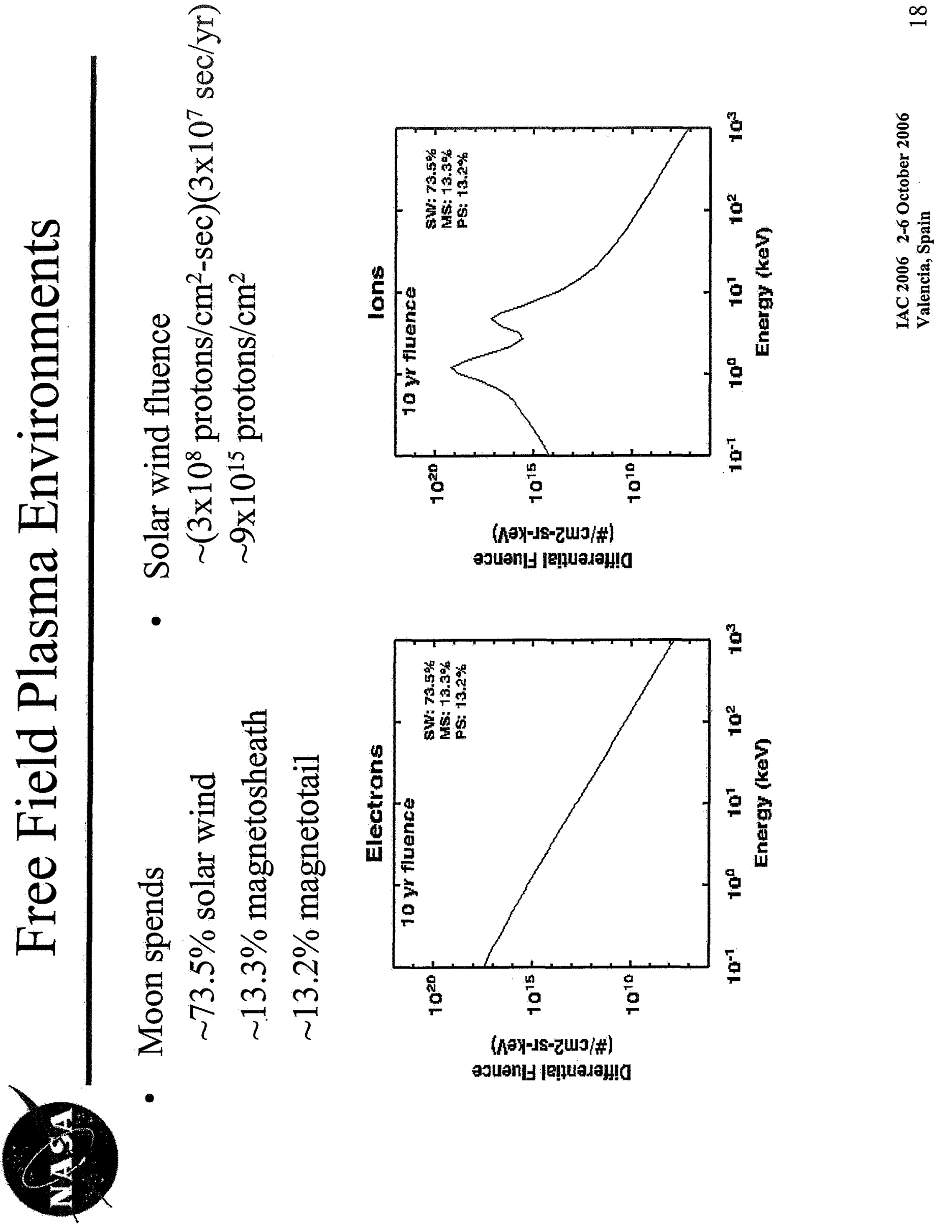




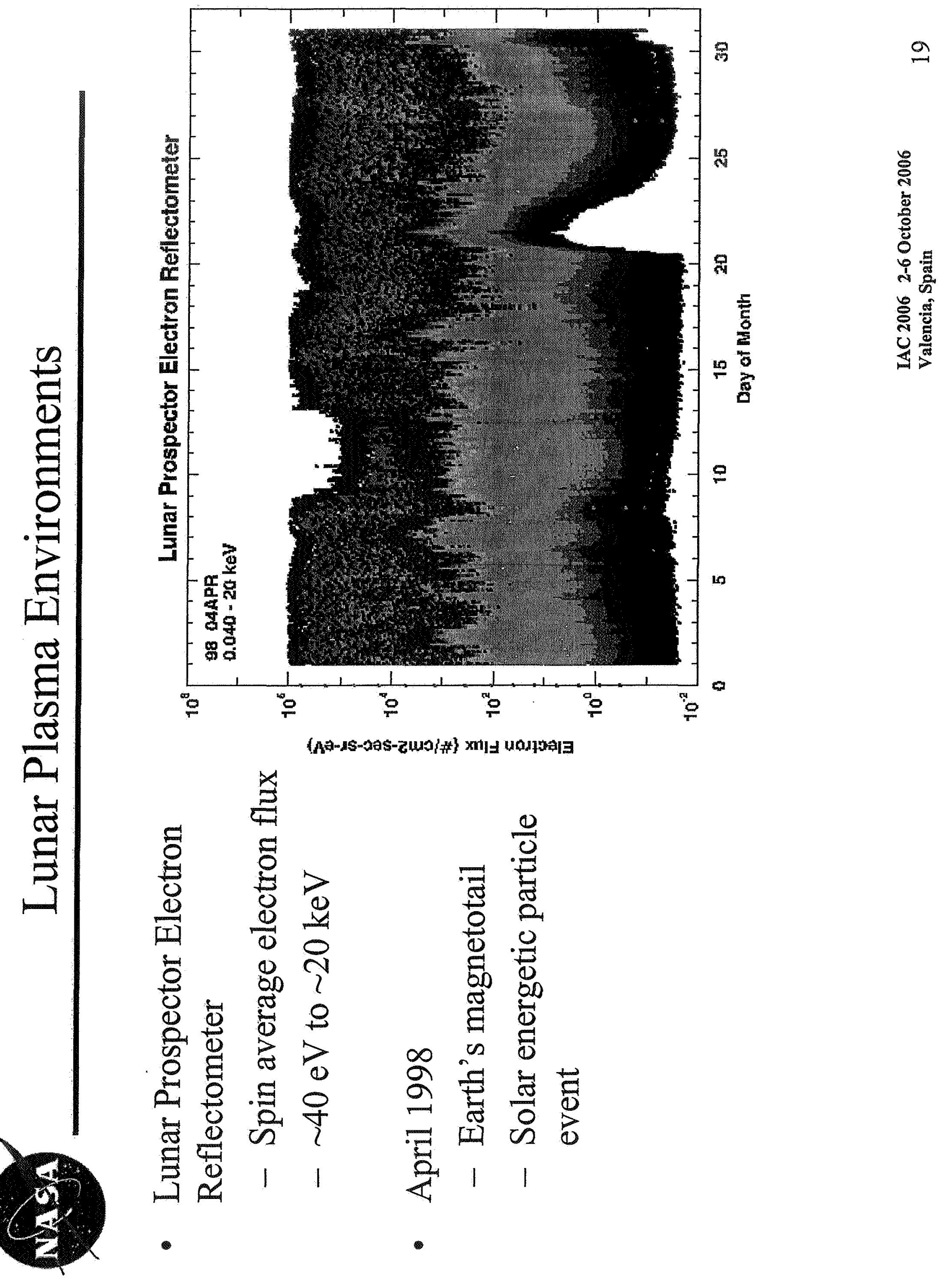




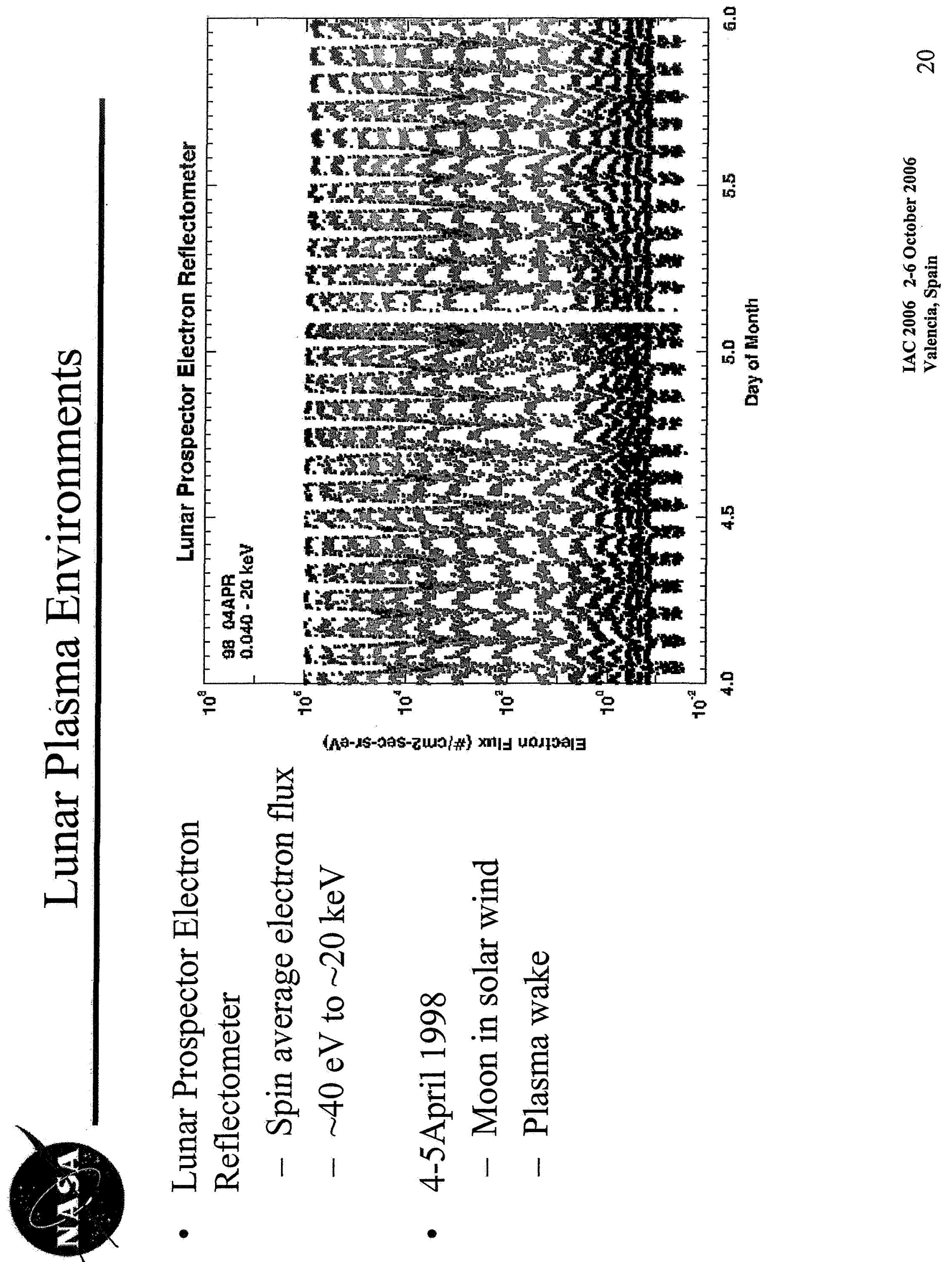




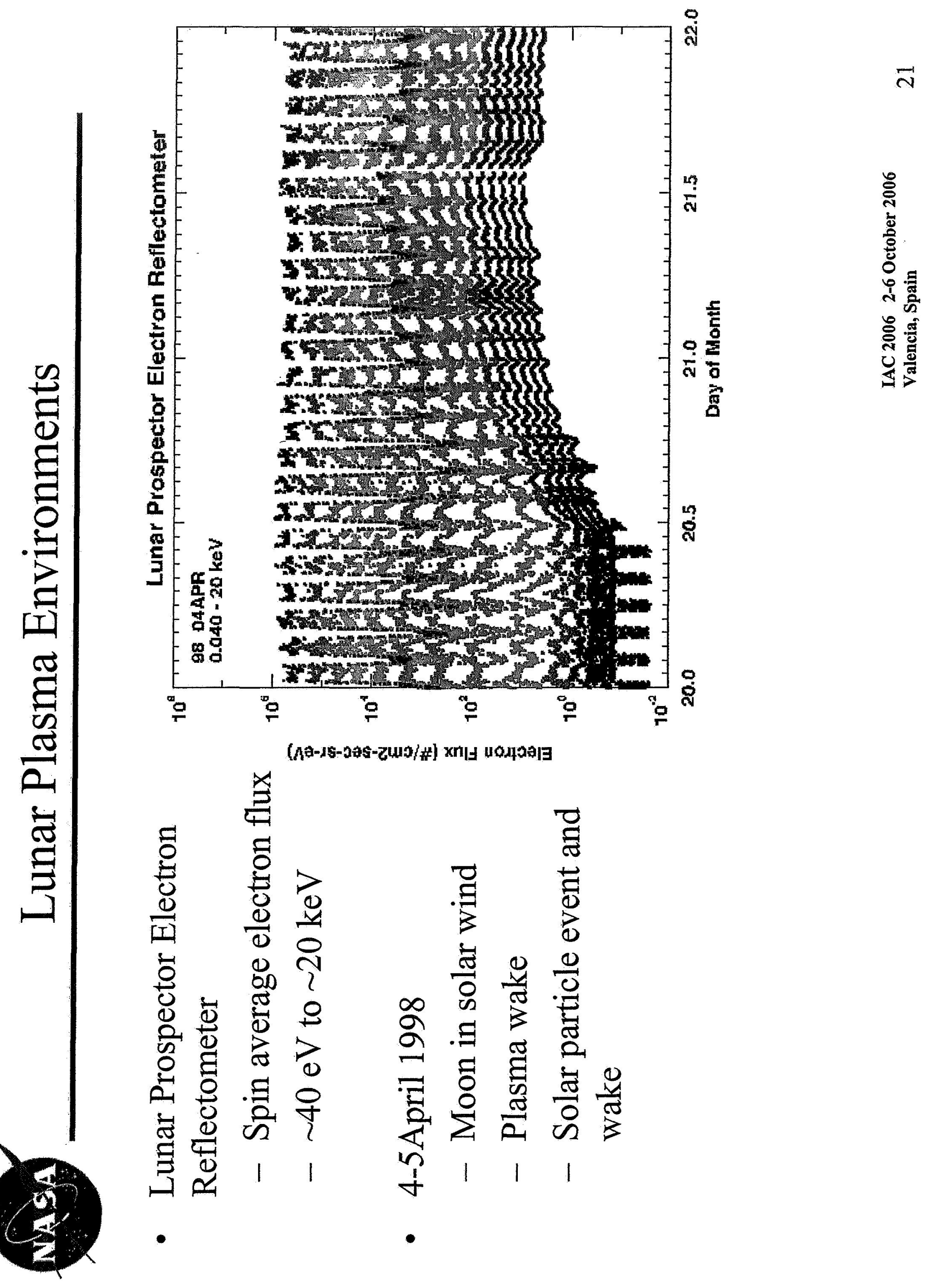




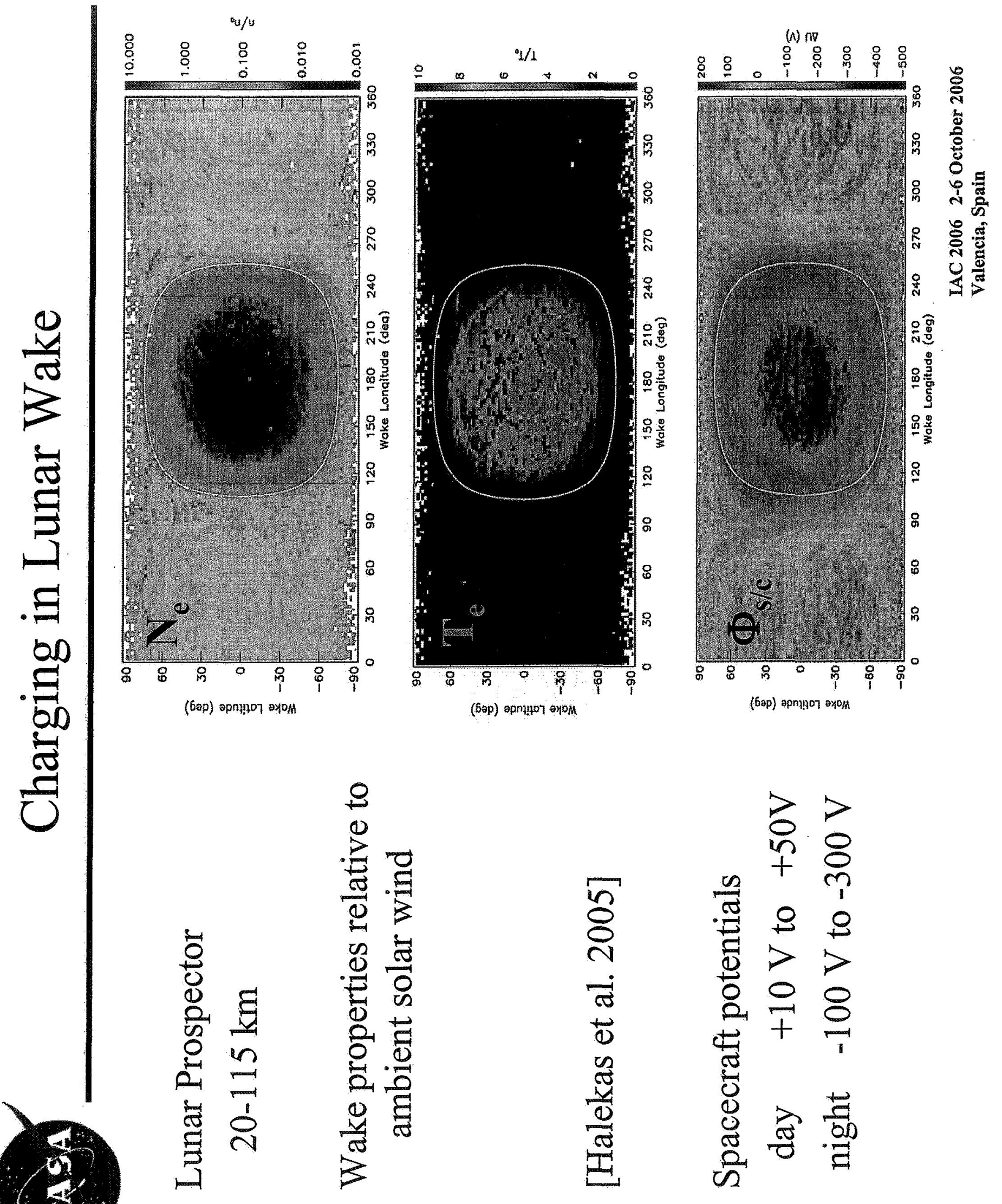




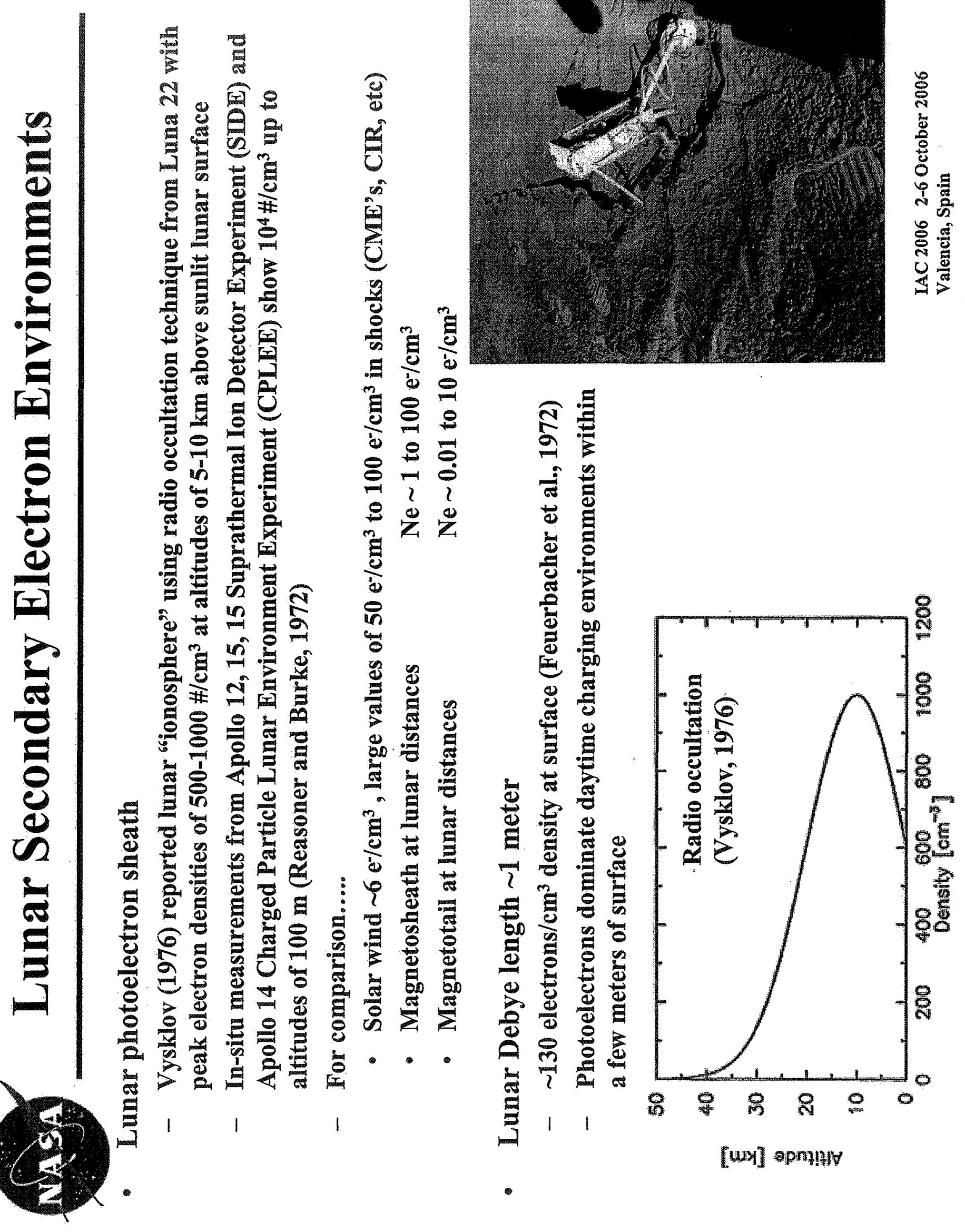




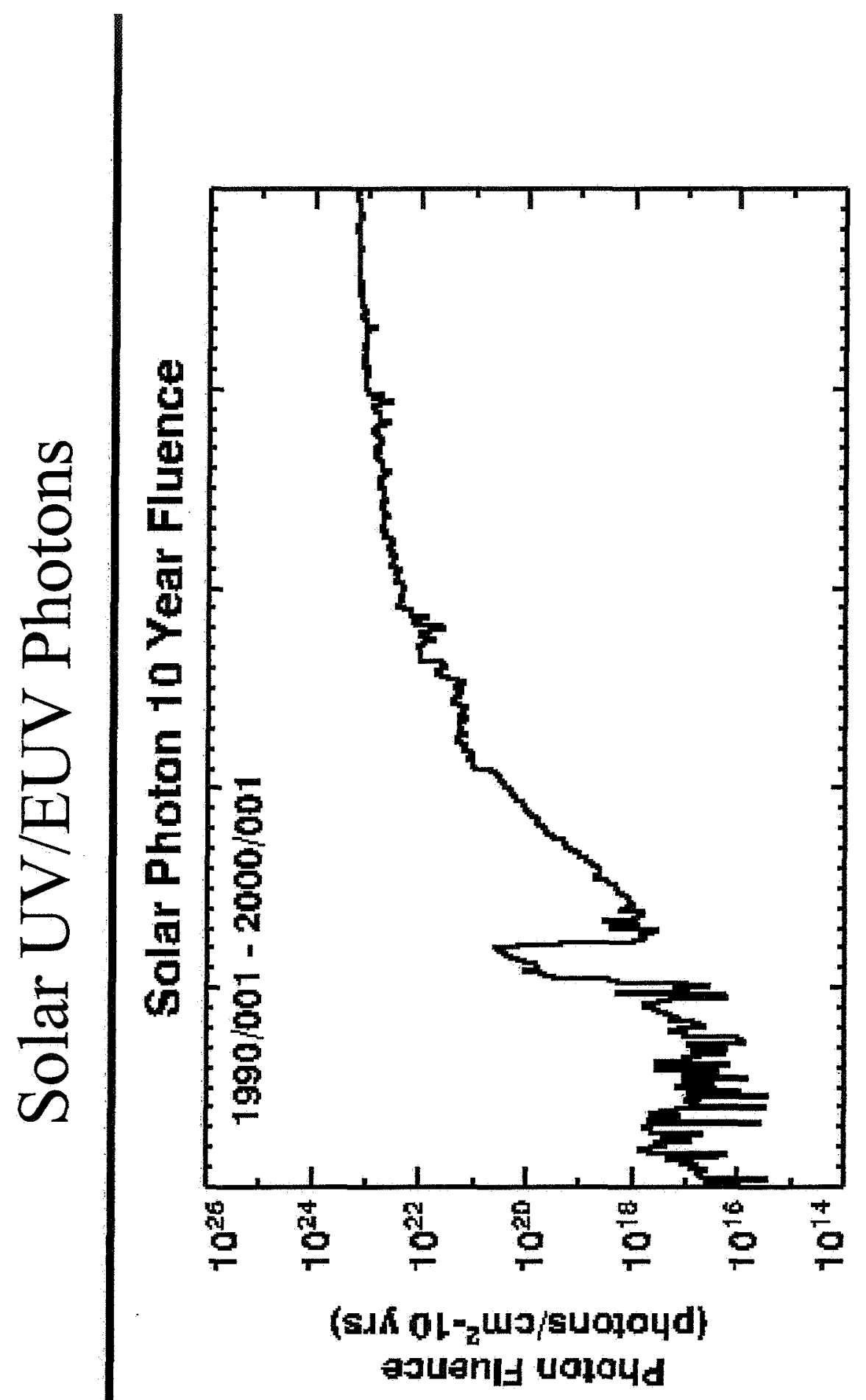

웅
뭉

莽

$\frac{8}{\frac{\pi}{8}}$ ปे

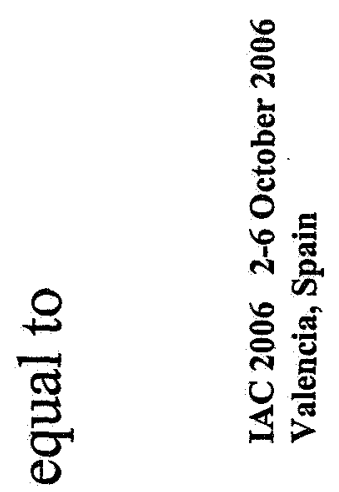

9 듬

$=\quad \stackrel{5}{2}$ \&

No क0

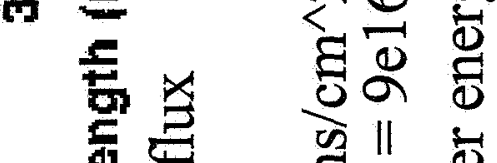

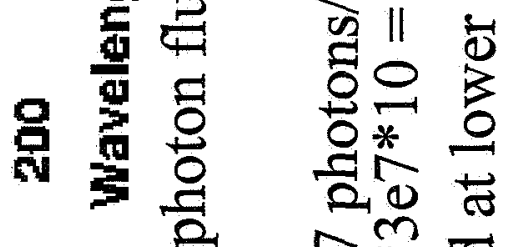

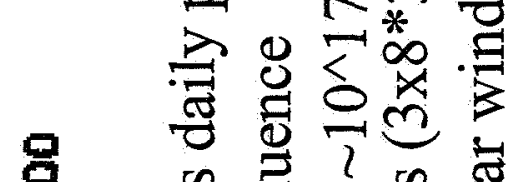

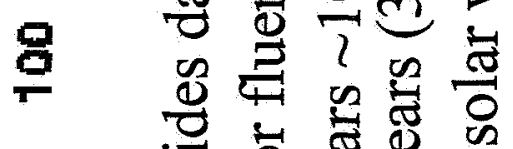

-

늄

- 용ㅎㅇ

局

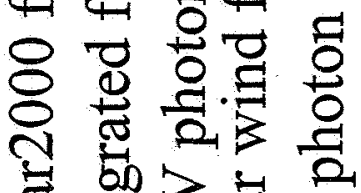

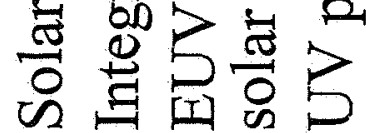


2
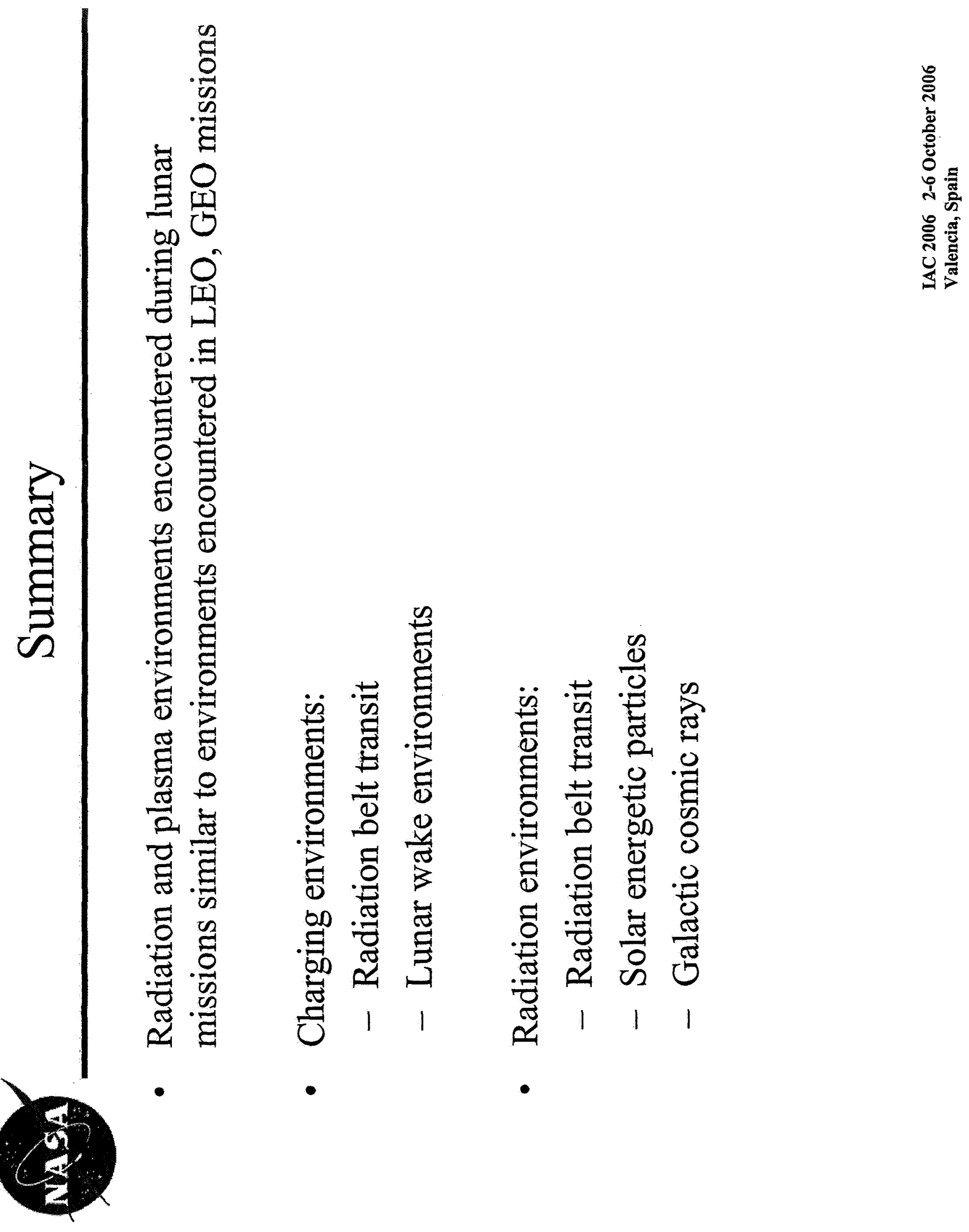